\title{
Signal Extraction and Automated Polarization Analysis of Multicomponent Array Data
}

\author{
by K. De Meersman,* M. van der Baan, and J.-M. Kendall ${ }^{\dagger}$
}

\begin{abstract}
We present a method to extract seismic signals from three-component array data and estimate their polarization properties at each station. The technique is based on a singular value decomposition (SVD) of the complex three-component analytic signal and applies to linearly as well as elliptically polarized seismic phases. To increase accuracy we simultaneously analyze data from different stations and apply a noise weighting based on prearrival data. For polarization analysis, an automated routine is also included. The automated routine selects the data window with the best signal-to-noise ratio from which to obtain a polarization. A linearity measure and a confidence interval accompany the polarization estimate at each station in the array. We test our technique for automated polarization analysis on synthetic $P$-wave data and compare results with those from other methods. A microseismic dataset from the North Sea provides a unique opportunity to statistically compare previous and independently obtained $P$-wave polarizations with those provided by the automated technique presented here. We conclude that, for $P$-wave polarization analysis, our method is robust and significantly more accurate than conventional, mainly manual methods. This is especially so on data with polarized and correlating background noise. It is also faster and provides meaningful quality estimates.
\end{abstract}

\section{Introduction}

Signal extraction and polarization analysis forms an important part of the processing and interpretation of multicomponent seismic data. There are many examples in seismology where polarization analysis helps with the identification and classification of different seismic phases (Flinn, 1965; Vidale, 1986; Mao and Gubbins, 1995; Earle, 1999). Knowing the polarization properties of seismic data also helps with the design of so-called polarization filters. These extract or attenuate signals with specific polarization properties (Montalbetti and Kanasewich, 1970; Bataille and Chiu, 1991; Reading et al., 2000; Du et al., 2000; de Franco and Musacchio, 2001) Polarization analysis is also central to the measurement of seismic anisotropy on $P$ waves (Bear et al., 1999; Schulte-Pelkum et al., 2001) and $S$ waves (Silver and Chan, 1991; Teanby et al., 2004). Finally, $P$-wave polarizations can provide estimates of the source azimuth and thereby help constrain earthquake locations (Maxwell et al., 1998; Dyer et al., 1999). Singular value decomposition (SVD), or eigen value decomposition (EVD), is generally

*Present address: Veritas GeoServices, 715 Fifth Avenue SW, Suite 2200, Calgary, Alberta, Canada, T2P 5A2; kristof_demeersman@ veritasdgc.com.

$\dagger$ Present address: Department of Earth Sciences, University of Bristol, Wills Memorial Building, Queen's Road, Bristol, BS8 1RJ, United Kingdom. used to estimate polarization properties and extract signal from data. Both techniques are closely related matrix operations. SVD applies to rectangular matrices, whereas EVD applies to square matrices. In early applications the threecomponent seismograms would be stored in a $N$ by 3 matrix, with $N$ the number of samples (Flinn, 1965; Montalbetti and Kanasewich, 1970). This time-domain approach suffers from stability problems in short time windows around zero crossings. A further restriction is that this approach can only deal with linearly polarized phases. Replacing the seismic data by their Fourier transforms, wavelet transforms, or analytic signals allows for the analysis of phases with linear and elliptical polarizations. Fourier and wavelet methods are best to deal with interfering seismic phases with different frequency content (Samson, 1973; Du et al., 2000). Analytic signals permit the use of short analysis windows (Vidale, 1986). This is ideal when dealing with multiple shortduration arrivals that are closely separated in time and have similar frequency content.

Various authors have stacked information from different three-component stations within an array (Jurkevics, 1988; Bataille and Chiu, 1991; Earle, 1999). This improves signal and polarization estimates because it increases the signal-tonoise ratio. The method is nevertheless restricted to (small) arrays over which signal and polarization properties remain 
constant. On the other hand it is possible to combine the $N$ data samples from $k$ three-component stations into one large $N$ by $3 k$ data matrix. SVD and EVD equally apply to these large data matrices. This approach can handle polarization variations within the array, and also benefits from an increased signal-to-noise ratio (Bear et al., 1999).

EVD and SVD usually rely on the assumption that the background noise is random and isotropic. A biased signal and polarization estimate is obtained in cases where these assumptions break down. Noise-weighted EVD and SVD can counter the negative effects of polarized background noise (Samson, 1983a, b; Du et al., 2000).

We present an automated time domain, weighted threecomponent array technique for robust polarization estimation. SVD and EVD are central to our approach, and following Vidale (1986), we use the analytic signal. We have combined this with multistation array analysis and noise weighting as per Bear et al. (1999) and Samson (1983a). The technique is applicable to array and repeat source data that are contaminated with coherent and polarized noise. The analysis window is automatically and iteratively optimized by identifying those samples with large deviations from the measured polarization and excluding them from the improved analysis window in the next iteration.

Next, we discuss the three-component approach of Flinn (1965) and then gradually add the components that make up our method for signal extraction and polarization analysis. The automated polarization analysis is introduced afterwards. We then apply the technique to both a synthetic and real $P$-wave dataset and discuss the results. The real data come from a microseismic experiment in the North Sea Valhall oil field conducted in 1998. The examples cover three main issues: (1) estimation and correction for relative arrival-time differences between the different three-component datasets, (2) the derivation of polarization uncertainty estimates and other quality control parameters, and (3) evaluation of accuracy through comparisons with the exact polarization estimates for the synthetic example and manually obtained estimates for the real dataset.

\section{Singular Value Decomposition and Eigen Value Decomposition}

\section{General Background}

Given a three-component (3C) seismic dataset with the seismograms $n(t), e(t)$, and $z(t)$ recorded in the north, east, and vertical directions, we can construct a $N$ by 3 data matrix $\mathbf{D}$ in which each column represents a seismogram over the time window $t \in\left[T_{1}, T_{N}\right]$, or $\mathbf{D}=[n(t), e(t), z(t)]$. SVD and EVD are popular techniques to find the waveform $\mathbf{u}_{S}$, magnitude $\sigma_{S}$, and polarization $\mathbf{v}_{S}$ of the signal that is present in the data $\mathbf{D}$. The SVD of the data in $\mathbf{D}$ is given by

$$
\mathbf{D}=\mathbf{U} \Lambda \mathbf{V}^{T}=\sum_{i=1}^{3} \sigma_{i} \mathbf{u}_{i} \mathbf{v}_{i}^{T}
$$

where $\mathbf{D}$ is the product of the $N$ by 3 matrix $\mathbf{U}$, the 3 by 3 diagonal matrix $\Lambda$, and the transpose $(T)$ of the 3 by 3 matrix $\mathbf{V}$. The unit left and right singular vectors $\mathbf{u}_{i}$ and $\mathbf{v}_{i}$ are the three column vectors of $\mathbf{U}$ and $\mathbf{V}$, respectively. The singular values $\sigma_{\mathrm{i}}$ are the diagonal elements of $\Lambda$. By convention they are ordered such that $\left|\sigma_{1}\right| \geq\left|\sigma_{2}\right| \geq\left|\sigma_{3}\right| \geq 0$.

The left singular vectors $\mathbf{u}_{i}$ and right singular vectors $\mathbf{v}_{i}$ are mutually orthogonal, or $\mathbf{U}^{T} \mathbf{U}=\mathbf{I}$ and $\mathbf{V} \mathbf{V}^{T}=\mathbf{I}$, where $\mathbf{I}$ is the identity matrix. An SVD therefore decomposes the data matrix $\mathbf{D}$ into its orthogonal parts $\sigma_{i} \mathbf{u}_{i} \mathbf{v}_{i}^{T}$. For 3C seismic data $\sigma_{i} \mathbf{u}_{i}$ represents the seismogram that is recorded by the $3 \mathrm{C}$ receiver in the direction of the polarization vector $\mathbf{v}_{i}$. It holds that $\sigma_{i} \mathbf{u}_{i}=\mathbf{D} \mathbf{v}_{i}$. The total seismic energy in the polarization direction $\mathbf{v}_{i}$ and over the interval $\left[T_{1}, T_{N}\right]$ is given by $\sigma_{i}^{2}$. It is a property of SVD that the polarization vectors $\mathbf{v}_{i}$ give the directions of maximal $\sigma_{1}^{2}$, intermediate $\sigma_{2}^{2}$, and minimal $\sigma_{3}^{2}$ seismic energy.

EVD is the decomposition of the data-correlation matrix $\mathbf{D}^{T} \mathbf{D}$ into its eigenvalues and eigenvectors, yielding

$$
\mathbf{D}^{T} \mathbf{D}=\mathbf{V} \Lambda^{2} \mathbf{V}^{T}=\sum_{i=1}^{3} \mathbf{v}_{i} \lambda_{i} \mathbf{v}_{i}^{T} .
$$

This definition follows immediately from (1). The squared singular values give the eigenvalues $\lambda_{i}=\sigma_{i}^{2}$. The right singular vectors $\mathbf{v}_{i}$ are the eigenvectors of $\mathbf{D}^{T} \mathbf{D}$ and the left singular vectors $\mathbf{u}_{i}$ are the eigenvectors of $\mathbf{D D}^{T}$.

Influence of Isotropic Random Noise. There are several important assumptions to consider when using SVD or EVD to estimate the true signal $\sigma_{S} \mathbf{u}_{S}$ and polarization $\mathbf{v}_{S}$ from data D. The signal must be linearly polarized, and the noise must be isotropic and uncorrelated with the signal. Isotropic noise is not polarized and its energy is equal in all directions. If we assume that $\mathbf{D}=\mathbf{S}+\mathbf{N}$, with $S=\sigma_{S} \mathbf{u}_{S} \mathbf{v}_{S}^{T}$ the true $3 \mathrm{C}$ signal and $\mathbf{N}$ the $3 \mathrm{C}$ noise, then we can rewrite (2) and obtain

$$
\mathbf{D}^{T} \mathbf{D}=\mathbf{S}^{T} \mathbf{S}+\mathbf{N}^{T} \mathbf{N}=\mathbf{v}_{S} \lambda_{S} \mathbf{v}_{S}^{T}+\lambda_{N} \mathbf{I}
$$

The noise correlation matrix $\mathbf{N}^{T} \mathbf{N}$ for isotropic random noise is simply the product of the identity matrix $\mathbf{I}$ with the noise energy in any direction $\lambda_{N}$. The first eigenvector $\mathbf{v}_{1}$ of the data-correlation matrix $\mathbf{D}^{T} \mathbf{D}$ defines the direction of maximum energy in the data. This is automatically the signal polarization and $\mathbf{v}_{1}=\mathbf{v}_{S}$. The total energy in this direction is $\lambda_{1}=\sigma_{1}^{2}=\lambda_{s}+\lambda_{N}$, with $\lambda_{1}>\lambda_{2}=\lambda_{3}=\lambda_{N}$. Since $\mathbf{D}=\mathbf{S}+\mathbf{N}$ we find that the waveform $\sigma_{1} \mathbf{u}_{1}=\mathbf{D} \mathbf{v}_{1}=(\mathbf{S}$ $+\mathbf{N}) \mathbf{v}_{1}$ is composed of the signal $\sigma_{S} \mathbf{u}_{S}=\mathbf{S} \mathbf{v}_{1}$ and the part of the random background $\mathbf{N} \mathbf{v}_{1}$. The signal-to-noise ratio (SNR) of this signal estimate $\sigma_{1} \mathbf{u}_{1}$ is thus

$$
\mathrm{SNR}=\frac{\lambda_{S}}{\lambda_{N}}=\frac{\lambda_{1}-\lambda_{2}}{\lambda_{2}}
$$

Influence of Nonisotropic Noise. The assumption of uncorrelated isotropic background noise is unrealistic for most 
seismic applications. If the noise is polarized but uncorrelated with the signal then $\mathbf{N}^{T} \mathbf{N} \neq \lambda_{N} \mathbf{I}$, but still $\mathbf{S}^{T} \mathbf{N}=0=$ $\mathbf{N}^{T} \mathbf{S}$. Expression (3) for the data-correlation matrix $\mathbf{D}^{T} \mathbf{D}$ becomes

$$
\mathbf{D}^{T} \mathbf{D}=\mathbf{S}^{T} \mathbf{S}+\mathbf{N}^{T} \mathbf{N}=\mathbf{v}_{S} \lambda_{S} \mathbf{v}_{S}^{T}+\sum_{i=1}^{3} \mathbf{v}_{N_{i}} \lambda_{N_{i}} \mathbf{v}_{N_{i}}^{T} .
$$

The noise correlation matrix $\mathbf{N}^{T} \mathbf{N}$ depends on the noise eigenvectors $\mathbf{v}_{N_{i}}$ and the noise eigenvalues $\lambda_{N_{i}}$ with $\lambda_{N_{1}} \geq$ $\lambda_{N_{2}} \geq \lambda_{N_{3}} \geq 0$. In general, the first eigenvector $\mathbf{v}_{1}$ of the data-correlation matrix $\mathbf{D}^{T} \mathbf{D}$ and the signal polarization vector $\mathbf{v}_{S}$ will be different. The angle between both vectors is typically a function of the noise and signal polarizations and of the ratios between signal and noise eigenvalues (Souriau and Veinante, 1975; Bataille and Chiu, 1991).

We illustrate the problems with polarized noise in Figure 1. For simplicity we use a two-component example, but the inferences made are true for any number of components. Correlation matrices are square and symmetric and have positive eigenvalues. They can be represented graphically as ellipses (or ellipsoids for more dimensions), which are their geometric forms. Figure 1a (left) shows the geometric forms of the data-, signal-, and noise-correlation matrices, $\mathbf{D}^{T} \mathbf{D}$, $\mathbf{S}^{T} \mathbf{S}$, and $\mathbf{N}^{T} \mathbf{N}$ for synthetic data with isotropic noise. The signal is linearly polarized and its correlation matrix is represented by a line. The geometric form of the isotropic noisecorrelation matrix is a circle.

Figure 1a (right) displays the variation in signal-to-noise ratio $\mathrm{SNR}_{\theta}$ and data variance, or data energy, $\lambda_{\theta}$ as a function of azimuth. For each azimuth $\theta$ we can define a vector $\mathbf{v}_{\theta}=$ $[\cos (\theta), \sin (\theta)]^{T}$. The data energy is then $\lambda_{\theta}=\left|\mathbf{D} \mathbf{v}_{\theta}\right|^{2}$. The signal-to-noise ratio $\mathrm{SNR}_{\theta}=\frac{\left|\mathbf{S v}_{\theta}\right|^{2}}{\left|\mathbf{N v}_{\theta}\right|^{2}}$ is the ratio of signal energy to noise energy. Figure 1a (right) shows that for data with isotropic noise the signal polarization $\mathbf{v}_{S}$ coincides with the first eigenvector $\mathbf{v}_{1}$ and with the direction of maximal signal-to-noise ratio $\mathbf{v}_{\mathrm{SNR}}$. This $\mathbf{v}_{\mathrm{SNR}}$ is the $\mathbf{v}_{\theta}$ for a $\theta$ that maximizes $\mathrm{SNR}_{\theta}$. Note that $\max \left(\mathrm{SNR}_{\theta}\right)=\mathrm{SNR}$ in (4).

Figure $1 \mathrm{~b}$ gives the geometric forms of the correlation matrices and the signal-to-noise ratio $\mathrm{SNR}_{\theta}$ and datavariance $\lambda_{\theta}$ as a function of azimuth $\theta$ for the case where the noise is not isotropic. Contrary to the isotropic case we find that the first eigenvector of the data-correlation matrix $\mathbf{v}_{1}$, the signal polarization $\mathbf{v}_{S}$, and the direction of maximal signal-to-noise ratio $\mathbf{v}_{\mathrm{SNR}}$ are all different. Therefore, the first left and right singular vectors $\mathbf{u}_{1}$ and $\mathbf{v}_{1}$ provide poor estimates for the signal $\sigma_{S} \mathbf{u}_{S}$ and its polarization $\mathbf{v}_{S}$. We also point out that the isotropic definition of the signal-to-noise ratio SNR in (4) no longer holds.

\section{Weighted Decompositions}

It is possible to correct for the presence of polarized random noise by applying a weighting to the data $\mathbf{D}$ (Samson, 1983a,b). We define the weighted data matrix $\mathbf{D}^{w}=$

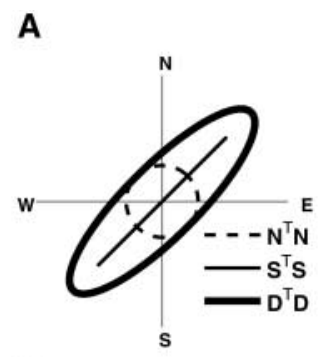

B

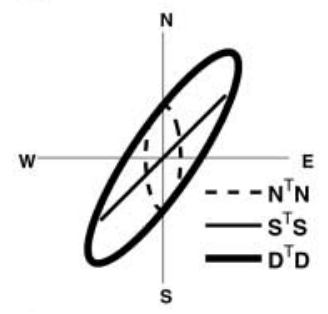

C

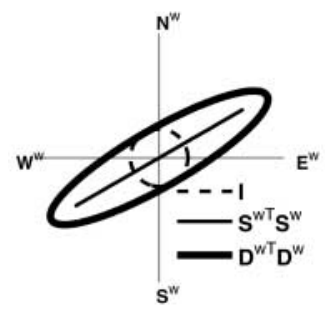

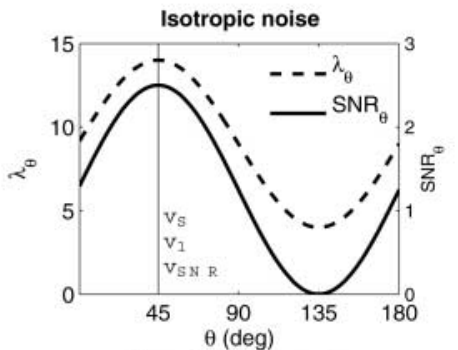
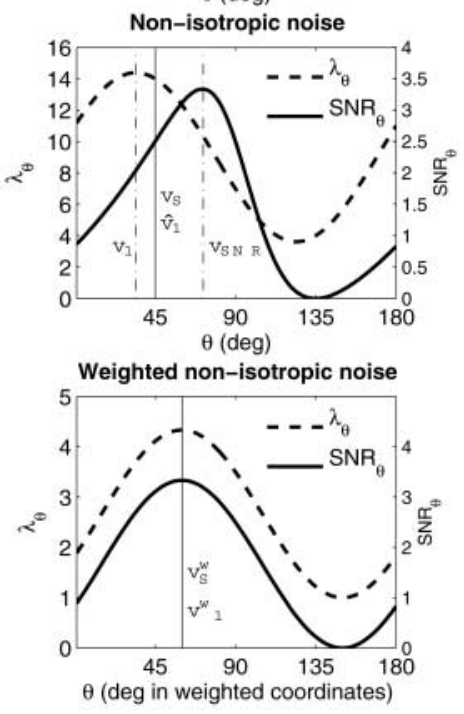

Figure 1. (Left) Geometric forms of the data-, signal-, and noise-correlation matrices, $\mathbf{D}^{T} \mathbf{D}$ and $\mathbf{S}^{T} \mathbf{S}$ respectively, and $\mathbf{N}^{T} \mathbf{N}$ for a 2D example. (Right) The signal-to-noise ratio $\mathrm{SNR}_{\theta}$ and data-variance $\lambda_{\theta}$ as a function of azimuth $\theta$. (a) Isotropic random noise and linearly polarized signal. The major axis of the datacorrelation ellipse, $\mathbf{v}_{1}$, is parallel to the signal polarization vector $\mathbf{v}_{S}$ and the direction of maximal signalto-noise ratio $\mathbf{v}_{\mathrm{SNR}}$. (b) Nonisotropic random noise and linearly polarized signal without weighting. The major axis of the data-correlation ellipse $\mathbf{v}_{1}$ is different from the signal polarization vector $\mathbf{v}_{S}$ and the direction of maximal signal-to-noise ratio $\mathbf{v}_{\mathrm{SNR}}$. (c) Example $b$ after weighting. The major axis of the weighted data-correlation ellipse $\mathbf{v}_{1}^{w}$ is parallel to the signal polarization vector $\mathbf{v}_{S}^{w}$. The vectors $\mathbf{v}_{\mathrm{SNR}}$ and $\hat{\mathbf{v}}_{1}$ can be derived from $\mathbf{v}_{1}^{w}$ and $\mathbf{W}=\mathbf{N}^{T} \mathbf{N}$.

$\mathbf{D} \mathbf{W}^{-0.5}=(\mathbf{S}+\mathbf{N}) \mathbf{W}^{-0.5}$ and its correlation matrix $\mathbf{D}^{w T} \mathbf{D}^{w}$ for a weighting matrix $\mathbf{W}=\mathbf{N}^{T} \mathbf{N}$. When SVD (1) and EVD (2) are applied to the weighted data and its correlation matrix we get

$$
\mathbf{D}^{w}=\mathbf{U}^{w} \Lambda^{w} \mathbf{V}^{w T}=\sum_{i=1}^{3} \mathbf{u}_{i}^{w} \sigma_{i}^{w} \mathbf{v}_{i}^{w T}
$$

and

$$
\mathbf{D}^{w T} \mathbf{D}^{w}=\sum_{i=1}^{3} \mathbf{v}_{i}^{w} \lambda_{i}^{w} \mathbf{v}_{i}^{w T}
$$


From this point we will use the superscript $w$ to identify vectors $\mathbf{v}_{i}^{w}$ and $\mathbf{u}_{i}^{w}$, and values $\sigma_{i}^{w}$ and $\lambda_{i}^{w}$ that result from SVD and EVD on weighted data $\mathbf{D}^{w}$. Similarly to (3) and (5), we can now rewrite the weighted data-correlation matrix $\mathbf{D}^{w T} \mathbf{D}^{w}$ as

$$
\mathbf{D}^{w T} \mathbf{D}^{w}=\mathbf{W}^{-0.5} \mathbf{S}^{T} \mathbf{S} \mathbf{W}^{-0.5}+\mathbf{I}=\mathbf{v}_{S}^{w} \lambda_{S}^{w} \mathbf{v}_{S}^{w T}+\mathbf{I} .
$$

This expansion demonstrates how the weighting has effectively transformed the polarized noise into isotropic noise, since $\mathbf{I}=\mathbf{W}^{-0.5} \mathbf{N}^{T} \mathbf{N} \mathbf{W}^{-0.5}$. Weighting affects the signal differently and the weighted signal $\mathbf{S}^{w}=\mathbf{S} \mathbf{W}^{-0.5}$ will have a different polarization and amplitude. This follows from $\mathbf{S}^{w}=\sigma_{S} \mathbf{u}_{S} \mathbf{v}_{S}^{T} \mathbf{W}^{-0.5}=\sigma_{S}^{w} \mathbf{u}_{S}^{w} \mathbf{v}_{S}^{w T}$. Here, the unit vector $\mathbf{v}_{S}^{w}$ is the weighted signal polarization and $\sigma_{S}^{w}=\sigma_{S}\left|\mathbf{v}_{S}^{T} \mathbf{W}^{-0.5}\right|$ is the weighted signal amplitude. The signal remains unchanged such that $\mathbf{u}_{S}=\mathbf{u}_{S}^{w}$. As the noise in the weighted data has now become isotropic, it will align with the direction of maximal weighted signal-to-noise ratio $\mathbf{v}_{\mathrm{SNR}}^{w}$ and the first eigenvector $\mathbf{v}_{1}^{w}$ with the polarization vector $\mathbf{v}_{S}^{w}$ of the weighted true signal (Fig. 1c). The signal-to-noise ratio of the weighted signal estimate $\sigma_{1}^{w} \mathbf{u}_{1}^{w}$ is found similarly to (4) and yields

$$
\mathrm{SNR}^{w}=\frac{\lambda_{1}^{w}-\lambda_{2}^{w}}{\lambda_{2}^{w}}
$$

Equation (6) and the property that $\mathbf{D}=\mathbf{D}^{w} \mathbf{W}^{0.5}$ lead to a new decomposition for the data matrix

$$
\mathbf{D}=\sum_{i=1}^{3} \mathbf{u}_{i}^{w} \sigma_{i}^{w} \mathbf{v}_{i}^{w T} \mathbf{W}^{0.5}=\sum_{i=1}^{3} \hat{\mathbf{u}}_{i} \hat{\sigma}_{i} \hat{\mathbf{v}}_{i}^{T}
$$

We use the unit vectors $\hat{\mathbf{u}}_{i}$ and $\hat{\mathbf{v}}_{i}$ to represent the waveforms and the polarizations of the signals in the data $\mathbf{D}$. Their amplitudes $\hat{\sigma}_{i}$ are equal to $\sigma_{i}^{w}\left|\mathbf{u}_{i}^{w} \mathbf{W}^{0.5}\right|$. The polarization vectors $\hat{\mathbf{v}}_{i}$ are not mutually orthogonal and different from the vectors $\mathbf{v}_{i}$ in (1). The same is true for the amplitudes $\hat{\sigma}_{i} \neq \sigma_{i}$. The signal vectors $\hat{\mathbf{u}}_{i}$ are orthogonal, but nevertheless different from the vectors $\mathbf{u}_{i}$ in (1). For completeness, (10) also leads to a new decomposition for the data-correlation matrix

$$
\mathbf{D}^{T} \mathbf{D}=\sum_{i=1}^{3} \mathbf{W}^{0.5} \mathbf{v}_{i}^{w} \lambda_{i}^{w} \mathbf{v}_{i}^{w T} \mathbf{W}^{0.5}=\sum_{i=1}^{3} \hat{\mathbf{v}}_{i} \hat{\lambda}_{i} \hat{\mathbf{v}}_{i}^{T} .
$$

For signal and polarization estimation on data with nonisotropic noise these decompositions are superior to SVD (1) and EVD (2). Earlier in Figure 1c we discussed that the first weighted right eigenvector $\mathbf{v}_{1}^{w}$ is identical with the weighted signal polarization $\mathbf{v}_{S}^{w}$. The accompanying weighted signal estimate $\sigma_{1}^{w} \mathbf{u}_{1}^{w}$ of the true weighted signal $\sigma_{S}^{w} \mathbf{u}_{S}^{w}$ has a maximal signal-to-noise ratio. Because, $\hat{\sigma}_{i} \hat{\mathbf{u}}_{i} \hat{\mathbf{v}}_{i}^{T}=$ $\sigma_{1}^{w} \mathbf{u}_{1}^{w} \mathbf{v}_{1}^{w T} \mathbf{W}^{0.5}$ it follows that $\hat{\mathbf{v}}_{1}$ gives the true signal polarization $\mathbf{v}_{S}$ and $\hat{\sigma}_{1} \hat{\mathbf{u}}_{1}$ is the estimate for the true signal $\sigma_{S} \mathbf{u}_{S}$ with highest possible signal-to-noise ratio as per (9).
To summarize, noise-weighted data decomposition provides us with the least noise biased estimate of the true signal and an accurate estimate of the true signal polarization. The decomposition itself is achieved in three main steps:

- Estimate the weighting matrix $\mathbf{W}$. In practice this is done by using prearrival seismic data and by assuming that the noise polarization properties are stationary over the length of the experiment. Previous efforts have shown that weighting is robust for small errors in the estimated noisecorrelation matrix (Samson, 1983; Du et al., 2000).

- Weight the data and determine the SVD of the weighted data $\mathbf{D}^{w}(10)$.

- Apply the inverse weighting matrix to the SVD of $\mathbf{D}^{w}$. This leads to the new decomposition for the original data $\mathbf{D}$, which is given in (10).

\section{Decompositions on Multiple Stations}

SVD and EVD naturally apply to data with any number of components and (6) and (7), or (10) and (11) can be generalized to deal with multiple $3 \mathrm{C}$ recordings simultaneously (Bear and Pavlis, 1999). Each of these individual 3C data matrices can contain information from a single event that is recorded on multiple receivers, or from repeat source data on the same receiver, or a combination of both. A new $N$ by $3 k$ data matrix $\mathbf{D}$ can then be generated by combining the $k$ individual $3 \mathrm{C}$ data matrices $\mathbf{D}^{j}$, with $j=1, \ldots, k$, such that $\mathbf{D}=\left[\mathbf{D}^{1}, \ldots, \mathbf{D}^{k}\right]$. We assume that the signal has identical waveforms $\mathbf{u}_{S}^{j}$ in each of the included datasets $\mathbf{D}^{j}$, but amplitudes $\sigma_{S}^{j}$ and polarizations $\mathbf{v}_{S}^{j}$ may vary. A moveout correction or time alignment between data from each $3 \mathrm{C}$ dataset is usually required. The main difference between the single and multistation approach is that the summation in equations (6), (7), (10), and (11) must now be done over $3 k$ instead of 3 and that the polarization vectors $\hat{\mathbf{v}}_{i}$ have $3 k$ rather than 3 elements. The new $3 k$ by $3 k$ weighting matrix $\mathbf{W}$ is calculated from an $N$ by $3 k$ matrix with prearrival data from all $3 \mathrm{C}$ stations.

The ultimate aim is to interpret the multistation estimates $\hat{\sigma}_{i} \hat{\mathbf{u}}_{i}$, and $\hat{\mathbf{v}}_{i}$, with $i=1,3, \ldots, k$, in terms of signals $\hat{\sigma}_{i}^{j} \hat{\mathbf{u}}_{i}$ and polarizations $\hat{\mathbf{v}}_{i}^{j}$ at the individual $j=1, \ldots, k 3 \mathrm{C}$ stations. To do this we break up the $3 k$-element polarization vectors $\hat{\mathbf{v}}_{i}$ into $k, 3$-element vectors $\hat{\mathbf{v}}_{i}^{j}$ for each station $j$. The values $\hat{\sigma}_{i}$ are also redistributed over the $k$ original stations such that $\hat{\sigma}_{i}^{j} \hat{\mathbf{v}}_{i}^{j}=\hat{\sigma}_{i}\left[\hat{v}(3 j-2)_{i}, \hat{v}(3 j-1)_{i}, \hat{v}(3 j)_{i}\right]^{T}$, with $\hat{v}(3 j)_{i}$ the $3 j$ th element of $\hat{\mathbf{v}}_{i}$. For each station this results in an alternative decomposition for the data matrix

$$
\mathbf{D}^{j}=\hat{\mathbf{U}} \hat{\Lambda}^{j} \hat{\mathbf{V}}^{j^{T}}=\sum_{i=1}^{3 k} \hat{\mathbf{u}}_{i} \hat{\sigma}_{i}^{j} \hat{\mathbf{v}}_{i}^{T}
$$

and for the data-correlation matrix

$$
\mathbf{D}^{j^{T}} \mathbf{D}^{j}=\hat{\mathbf{V}}^{j} \hat{\Lambda}^{j^{2}} \hat{\mathbf{V}}^{j^{T}}=\sum_{i=1}^{3 k} \hat{\mathbf{v}}_{i}^{j} \hat{\lambda}_{i}^{j} \hat{\mathbf{v}}_{i}^{j^{T}}
$$


Contrary to the EVD and SVD decompositions in (1) and (2), and the noise-weighted decompositions in (10) and (11), where the data in each station are the sum of three signals, the previous multistation decompositions view the data as the sum of $3 k$ different signals. The waveforms $\hat{\mathbf{u}}_{i}$ of these signals are the same for all stations, but their amplitudes $\hat{\sigma}_{i}^{j}$ and polarizations $\hat{\mathbf{v}}_{i}^{j}$ may vary.

The main advantage of this multistation approach is that it benefits from a potential $\sqrt{j}$-fold increase in the signal-tonoise ratio, without the need to assume identical signal polarizations at all stations. The latter assumption is made when stacking matrices. Even the influence of signalcorrelated noise will be reduced as long as this noise is not present in all stations, or out of phase. Obviously this leads to better estimates of the signal $\hat{\sigma}_{S}^{j} \hat{\mathbf{u}}_{S} \approx \hat{\sigma}_{1}^{j} \hat{\mathbf{u}}_{1}$ and their polarizations $\hat{\mathbf{v}}_{S}^{j} \approx \hat{\mathbf{v}}_{1}^{j}$.

Multistation data decompositions are achieved by combining different $3 \mathrm{C}$ data matrices of the same wave into one large data matrix. It is important that arrival-time differences between the individual 3C datasets are corrected for. After decomposition with (1) or (10) of the combined matrix, we simply break up the multistation polarization vectors into $3 \mathrm{C}$ polarization vectors that relate to the individual stations. This leads to a new decomposition (12) for each individual station, which consists of $3 k$ uncorrelated signals $\hat{\sigma}_{i}^{j} \hat{\mathbf{u}}_{i}^{j}$ with nonorthogonal polarizations $\hat{\mathbf{v}}_{i}^{j}$, where $k$ is the number of $3 \mathrm{C}$ stations in the analysis.

\section{Analytic Signals}

The use of time-domain signals limits us to analyzing linearly polarized signals only. Signals with elliptical polarizations can be analyzed with the use of analytic signals (Vidale, 1986). Linear polarizations are treated as a subset of elliptical polarizations with infinite ellipticity. The use of analytic signals also allows us to use shorter time windows or even compute instantaneous polarization attributes (Schimmel and Gallart, 2003). The single, multistation, weighted, or unweighted equations (1), (2), (10), (11), (12), and (13) can be used as before, but with the transpose (T) replaced by the complex conjugate transpose $(H)$. The analytic signals are formed by taking the original seismograms and adding their Hilbert Transform as the complex part. The new, complex data matrix $\mathbf{D}^{\varsigma}$ is then simply formed by replacing the real seismograms with the analytic signals. The same applies to the construction of the complex weighting matrix $\mathbf{W}^{\varsigma}$. Note that from this point onward we use the symbol $\varsigma$ to distinguish between real matrices and vectors and their complex counterparts.

If we take the complex data matrix $\mathbf{D}^{\varsigma}$ for the noiseweighted multistation case then the waveform estimates $\hat{\mathbf{u}}_{i}^{\varsigma j}$ and polarization vectors $\hat{\mathbf{v}}_{i}^{\varsigma j}$ will be complex, but the amplitudes $\hat{\sigma}_{i}^{\varsigma j}$ remain real. Each column in $\hat{\sigma}^{\varsigma j}{ }_{i} \hat{\mathbf{u}}^{\varsigma j}{ }_{i}^{j} \hat{\mathbf{v}}^{\zeta{ }_{i}}{ }_{i}^{H}$ is the product of the analytic signal $\hat{\sigma}^{\varsigma j}{ }_{i} \hat{\mathbf{u}}^{\subseteq j}{ }_{i}$ with an element of the complex polarization vector $\hat{\mathbf{v}}_{i}{ }_{i} H$. Typically these columns differ by a constant amplitude ratio and phase rotation. If we consider only the real part $\Re\left(\hat{\sigma}_{i}^{\varsigma j} \hat{\mathbf{u}}_{i}^{\varsigma j} \hat{\mathbf{v}}^{\varsigma j}{ }_{i}^{H}\right)$, then its columns are still scaled and phase-rotated copies of one another. This gives rise to elliptical particle motion and leads to the decomposition of $\Re\left(\mathbf{D}^{\varsigma}\right)$ into its elliptically polarised parts $\Re\left(\hat{\sigma}^{\zeta j} \hat{\mathbf{u}}_{i}{ }_{i}^{j} \hat{\mathbf{v}}^{\zeta{ }_{i}{ }^{H}}\right)$. The semimajor, $\mathbf{a}_{i}^{j}$, and semiminor, $\mathbf{b}_{i}^{j}$, axes of the polarization ellipse of each elliptical part result from a real SVD (1) on $\Re\left(\hat{\sigma}_{i}^{S j} \hat{\mathbf{u}}^{\zeta j}{ }_{i} \hat{\mathbf{v}}_{i}{ }_{i}^{H}\right)$. They can also be derived directly from the complex polarization vector, $\hat{\mathbf{v}}_{i}^{\varsigma j}$, as explained in Samson and Olsen (1980) and Vidale (1986).

\section{Automatic Estimation of Polarization}

The basis of our automated procedure for polarization analysis is a weighted multistation approach that uses the analytic signal. The automation deals mainly with the problem of selecting the optimal data window $t \in\left[T_{1}, T_{2}\right]$ over which the signal polarization is estimated. The aim is to produce good-quality polarizations with meaningful uncertainty estimates. The optimal analysis window should exclude all prearrival and postarrival noise, and samples contaminated by coherent noise with deviating polarization properties, such as secondary arrivals. This can be achieved by iteratively removing samples from the analysis window that produce unreasonably large-angle misfits relative to a measured provisional polarization. Individual samples can be removed because the use of analytic signals allows us to estimate instantaneous polarization attributes.

We define our sample misfit angles $\gamma(t)$ from the weighted data $\mathbf{D}^{\varsigma w}$ and its first left singular vector $\mathbf{v}_{1}^{\varsigma w}$.

$$
\gamma(t)=\arccos \frac{\left|\mathbf{d}^{\varsigma w H}(t) \mathbf{v}_{1}^{\varsigma w}\right|}{\left|\mathbf{d}^{\varsigma w}(t)\right|} .
$$

The $3 k$-element data-vector $\mathbf{d}^{\varsigma w}(t)$ represents one-time sample of the complex data and is a row vector of $\mathbf{D}^{\varsigma w}$. The complex matrix $\mathbf{D}^{\varsigma w}$ contains the moveout-corrected analytic signals from $k 3 \mathrm{C}$ stations. The $\gamma(t)$ values can vary between $0^{\circ}$ and $90^{\circ}$, and give the angles between each data vector and the provisional unit polarization vector $\mathbf{v}_{1}^{\varsigma w}$. The angle misfits are preferably derived from weighted data as weighting normalizes the noise contribution of different stations and receiver components, and makes the misfit angles $\gamma(t)$ more sensitive to the presence of coherent noise.

The misfit angles are used to define the spherical variance $v^{2}$, which is given by

$$
\begin{aligned}
& v^{2}=1-\sum_{t=T_{1}}^{T_{2}} w(t) \cos ^{2} \gamma(t) \\
& \text { where } w(t)=\frac{\left|\mathbf{d}^{\varsigma w}(t)\right|^{2}}{\sum_{t=T_{1}}^{T_{2}}\left|\mathbf{d}^{\varsigma w}(t)\right|^{2}}
\end{aligned}
$$

This definition varies slightly from Fisher et al. (2004) and Butler (1992) because the contribution from each sample is 
weighed according to its normalized energy $w(t)$, and because the data are complex. In practice, the spherical variance is computed from the eigenvalues $\lambda_{i}^{\varsigma w}$ (7) of the weighted data-correlation matrix $\mathbf{D}^{\varsigma w H} \mathbf{D}^{\varsigma w}$ by

$$
v^{2}=1-\frac{\lambda_{1}^{\varsigma w}}{\sum_{i=1}^{\text {all }} \lambda_{i}^{\varsigma w}} .
$$

For a confidence percentage $\alpha$ we define the interval [ $-\mu_{\alpha}$, $\left.+\mu_{\alpha}\right]$, in which $\alpha$ percent of the $\gamma(t)$ angles are expected to lie. After Fisher et al. (2004) we have

$$
\mu_{\alpha}=\arcsin e_{\alpha} v \text { and } e_{\alpha}=\sqrt{-\ln (1-\alpha)} .
$$

The optimal data window for polarization analysis is found by iteratively removing poor-quality samples from an initial data window. At each iteration, a provisionally array-based polarization vector $\mathbf{v}_{1}^{\varsigma w}$ is obtained with misfit angles $\gamma(t)$ for the samples used. Poor-quality samples are identified by misfit angles $\gamma(t)$ that lie outside the expectancy interval $\left[-\mu_{\alpha},+\mu_{\alpha}\right]$. This process is repeated until no outliers are identified, or until a minimum number of remaining samples is reached. Like the initial data window, the confidence percentage $\alpha$ and the minimum number of samples in the optimized window are user-defined parameters. We find the confidence percentage $\alpha$ is generally over $85 \%$ for adequately sampled data. The typical minimum number of samples is equivalent to halve or one dominant wavelength.

The optimized data window is usually irregularly sampled in time and contains the most consistent samples within the initial data window. Small changes in this initial data window are therefore unlikely to affect the final solution. Note that the statistics of our procedure use the $\gamma(t)$ values of an entire array. These array-based estimates are preferred over their station-based counterparts because they "average out" possible non-Gaussian and bimodal behavior of the angle misfits from individual stations. This benefits accuracy and robustness in the presence of polarised background noise.

\section{Practical Implementation on $P$-Wave Array Data}

The automated technique is applied in a three-stage procedure to $P$-wave events from a $3 \mathrm{C}$ receiver array (Fig. 2). The first stage obtains accurate relative $P$-wave arrival times between the different $3 \mathrm{C}$ receivers. The second stage is our automated polarization analysis. The final stage uses estimated linearities and uncertainties to perform quality control and to identify stations with poor results. Estimates from the identified bad stations are discarded and the complete threestage process is repeated for the remaining stations.

To avoid introducing artificial phase effects when estimating the signal and polarization, it is vital that the relative arrival times between stations are known accurately. We use

\section{Work flow for automated P-wave polarization analysis}

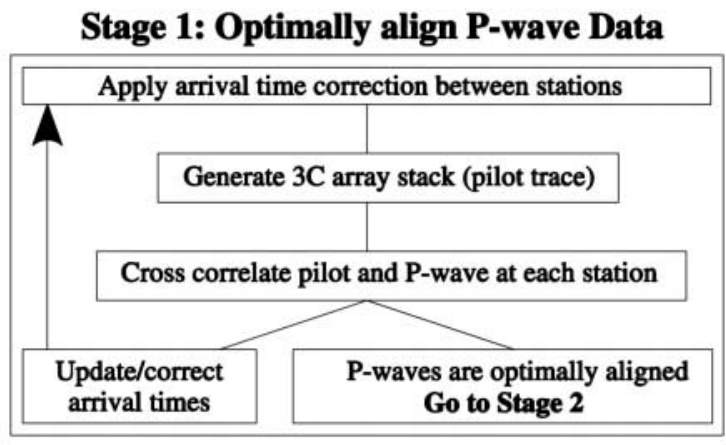

Stage 2: Polarization analysis

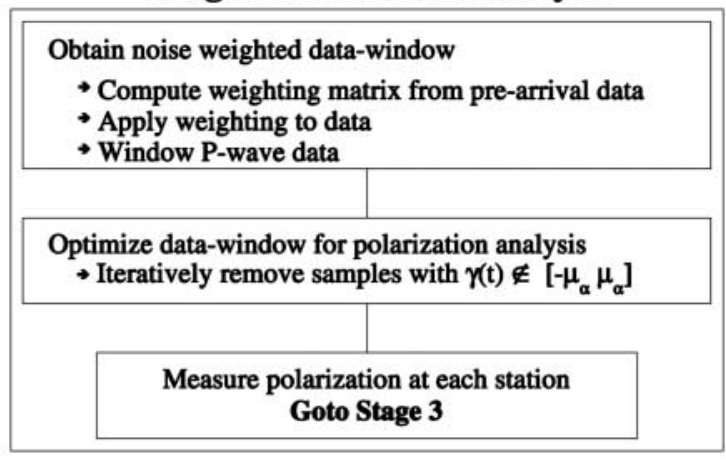

\section{Stage 3: Quality control}

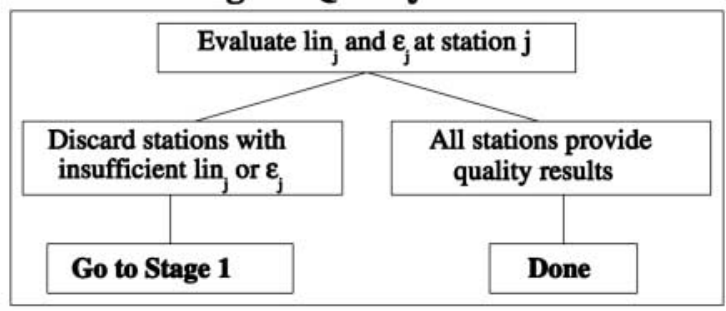

Figure 2. Flowchart of the processing procedure for automated $P$-wave polarization analysis on array data. A three-step procedure is followed, starting with the arrival-time alignment of data from different stations and followed by polarization analysis and quality control.

an iterative and coherency-based stack optimization technique similar to that of Rowe et al. (2002). Manually picked arrival times are used to generate a $3 \mathrm{C}$ stack called the pilot trace. Cross-correlation functions between the $3 \mathrm{C}$ pilot and the $3 \mathrm{C}$ data from each station provide $P$-wave arrival-time corrections at every station. A new $3 \mathrm{C}$ pilot trace is then created with updated arrival times. This process is repeated until the arrival-time corrections converge to zero. Conversion usually occurs quickly ( $\approx$ three iterations). It is best to use noise-weighted data $\mathbf{D}^{w}$ to minimize the negative effects of stations with high noise levels. 
In the final stage, a complex polarization estimate $\hat{\mathbf{v}}_{1}^{\varsigma j}$ at each $j$ th $3 \mathrm{C}$ station is obtained from (12). We take as the $P$-wave polarization, the semimajor axis $\mathbf{a}_{1}^{j}=$ $\left[a_{1}^{j}(1), a_{1}^{j}(2), a_{1}^{j}(3)\right]^{T}$ of the polarization ellipse defined by the complex polarization $\hat{\mathbf{v}}^{\varsigma j}{ }_{1}$. The $P$-wave polarization azimuth $\theta_{j}$ and inclination $\phi_{j}$ in each station are given by:

$$
\begin{aligned}
\theta_{j} & =\arctan \left(\frac{a_{1}^{j}(2)}{a_{1}^{j}(1)}\right), \\
\phi_{j} & =\arctan \left(\frac{\sqrt{a_{1}^{j^{2}}(2)+a_{1}^{j^{2}}(1)}}{a_{1}^{j}(3)}\right) .
\end{aligned}
$$

We introduce two useful quality-control parameters for $P$ wave analysis. The first is $P$-wave linearity $\operatorname{lin}_{j}$ and the second is a confidence interval $\left[-\varepsilon_{\alpha_{j}},+\varepsilon_{\alpha_{j}}\right]$ on the $P$-wave polarization, $\mathbf{a}_{1}^{j}$. After Claassen (2001) we define

$$
\operatorname{lin}_{j}=\left|\mathbf{a}_{1}^{j}\right| .
$$

The linearity $l i n_{j}$ is 1 for perfectly linearly polarized $P$ waves and decreases to 0.5 for $P$ waves with circular polarizations. For $P$ waves with high linearities we can relate the spherical variance $v^{2}$ in (16) to an uncertainty estimate for the $P$-wave polarization $\mathbf{a}_{1}^{j}$. If $N$ is the number of samples in the optimized analysis window, then the angular confidence interval $\left[-\varepsilon_{\alpha_{j}},+\varepsilon_{\alpha_{j}}\right]$ relates to the confidence percentage $\alpha$. Similar to Fisher et al. (2004) we have

$$
\varepsilon_{\alpha_{j}}=\arcsin \frac{e_{\alpha} v}{\sqrt{N}} \text { and where } e_{\alpha} \text { is defined in (17). }
$$

\section{Examples}

\section{Synthetic $P$-Wave Example}

The following tests on synthetic data compare our automated weighted multistation polarization analysis with unweighted and single-station methods. They also verify the use and reliability of the proposed quality-control parameters $\varepsilon_{\alpha_{j}}$ and $l i n_{j}$. The synthetic data are from a four-station $3 \mathrm{C}$ array with 1-msec sampling interval (Fig. 3). The $P$ wave is a $30-\mathrm{Hz}$ sine wave with exponential amplitude decay. $P$ wave energy and arrival times are kept constant between stations. Stations 3 and 4 are both contaminated with vertically polarized coherent noise. At station 3 this noise is a delayed $25-\mathrm{Hz}$ sine wave, and at station 4 it is a simultaneously arriving $30-\mathrm{Hz}$ cosine wave. Both waves have exponential amplitude decays and cause nonlinear particle motion. Random background noise is added to all stations. On stations 2 and 4 the energy level of this noise on the vertical component is increased so it becomes polarized. All other relevant signal and noise parameters are given in Table 1.
The noise is such that the signal-to-noise ratio decreases with station number.

In a first test we evaluate five alternative analysis methods by comparing polarization estimation errors with respect to the known signal polarizations. The confidence intervals $\left[-\varepsilon_{95_{j}},+\varepsilon_{95_{j}}\right]$ and linearities $\operatorname{lin}_{j}$ that accompany these polarization estimates are also considered. Table 2 provides the average test results from 200 realizations with different added random background noise. Note that the synthetic data in this test are optimally time aligned and therefore do not require a cross-correlation-based data alignment. Next is a summary of the five polarization analysis techniques used:

1. Single-station $3 \mathrm{C}$ approach with isotropic noise assumption as in equation (1) and similar to Vidale (1986).

2. Single-station 3C approach with noise weighting as in equation (10) and similar to Samson (1983b).

3. Multistation approach with noise weighting as in equation (12).

4. Noise-weighted single-station approach as in equation (10) and with window optimization applied.

5. Noise-weighted multistation approach as in equation (12) and with window optimization applied.

These techniques are tested on 150-msec-long (initial) data windows. In weighting, the weighting matrix is calculated from $100 \mathrm{msec}$ of simulated prearrival noise. An example of this prearrival noise is shown on Figure 3. Analysis methods that apply window optimization (methods 3 and 5) use an interval of $\left[-\mu_{90},+\mu_{90}\right]$ to identify bad-quality samples. The minimum number of samples in the optimized analysis window is 30 .

The estimation errors reported in Table 2 indicate significant variations in accuracy between the different techniques and stations. The data in station 1 are composed of signal and isotropic random noise and satisfy the requirements for 3C unweighted SVD. As expected, all tested methods accurately estimate the $P$-wave polarizations. The signal in station 2 is contaminated by polarized random background noise and satisfies the requirements for weighted analyses. The superiority of weighted approaches in this case is confirmed by comparing measurement errors from methods 1 and 2 in Table 2 . The typical error for any of the weighted methods is $0^{\circ}$ on average. It is $14.5^{\circ}$ for the unweighted $3 \mathrm{C}$ estimate. The data in station 3 are composed of signal and later-arriving correlated noise. Both weighted and unweighted methods cannot handle this situation if the entire 150 -msec analysis window is used. The most accurate polarization estimates are found with our window optimization procedure (methods 4 and 5 in Table 2). This successfully removes the secondary arrival from the analysis window. Station 4 records signal with polarized random background noise and simultaneously arriving coherent noise. None of the tested methods is expected to handle this situation perfectly. However, the errors on multistation polarizations $\left(\approx 7^{\circ}\right.$ for methods 3 and 5 ) are significantly smaller than 

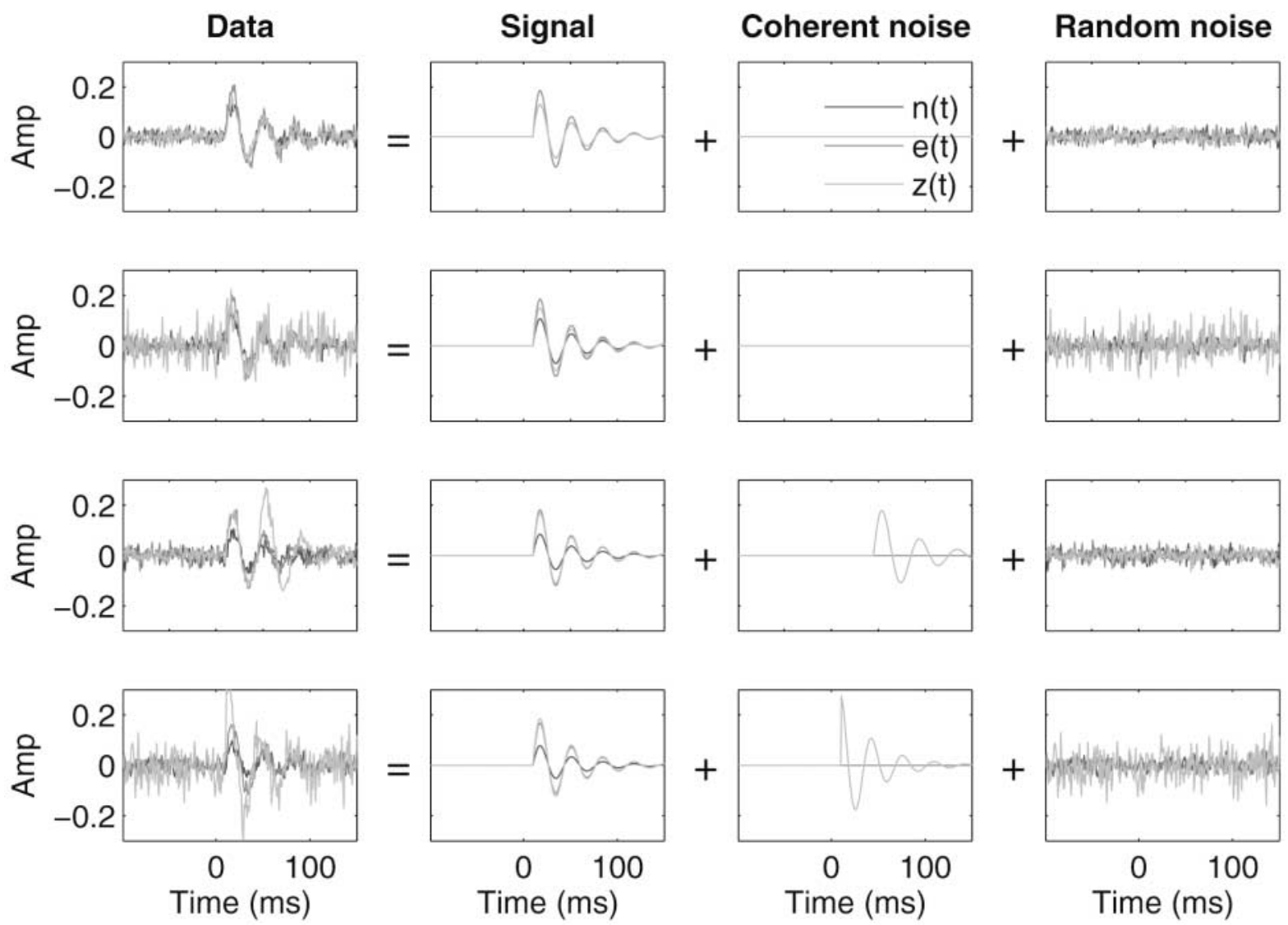

Figure 3. Synthetic 3C $P$-wave dataset from a four-station array. For each station and from left to right we show the data, its signal part, and its coherent and random noise parts. The signal is a $30-\mathrm{Hz}$ sine wave arriving at time $=0 \mathrm{msec}$. The coherent noise in station 3 arrives $35 \mathrm{msec}$ after the signal and is a vertically polarized $25-\mathrm{Hz}$ sine wave. The coherent noise on station 4 is a $30-\mathrm{Hz}$ cosine wave and arrives with the signal. Signal and coherent noise have exponential amplitude decay. Stations 2 and 4 are also contaminated with vertically polarized random background noise. More information on data properties can be found in Table 1 .

those on single-station estimates $\left(>16^{\circ}\right.$ for methods 1,2 , and 4). Linearities $\operatorname{lin}_{j}$ from single-station analyses (methods $1,2$, and 4$)$ are typically high ( $\geq 90 \%)$, yielding a misleading confidence in the estimations. In fact, only linearities from our method (5 in list) correctly indicate the accuracy of the polarization estimates. Similar observations can be made for the confidence intervals $\left[-\varepsilon_{95_{j}},+\varepsilon_{95_{j}}\right]$ that accompany the polarization estimates. These intervals are mainly a function of the signal-to-noise ratio at the station of interest and the number of samples used. The multistation approach best separates the signal from background noise and produces more reliable uncertainties for the polarizations. Singlestation polarization analyses underestimate the amount of noise in the data and, as a result, the uncertainty intervals.

To summarize we find that all tested methods provide similar and accurate results on good-quality data. On poorquality data with polarized noise we find that our technique is superior to any of the other tested methods. Our approach also provides the most reliable polarization linearity $\operatorname{lin}_{j}$ and confidence $\varepsilon_{95_{j}}$ estimates in any case.
Table 1

Signal and Noise Parameters for the Synthetic 3C Array $P$-Wave Dataset in Figure 3

\begin{tabular}{lrrrc}
\hline & \multicolumn{4}{c}{ Station Number } \\
\cline { 2 - 5 } Synthetic Data Properties & 1 & 2 & 3 & 4 \\
\hline Signal & 55 & 60 & 65 & 65 \\
$\quad$ Azimuth (deg) & 30 & 35 & 40 & 45 \\
$\quad$ Inclination (deg) & 1 & 1 & 1 & 1 \\
$\quad$ Energy & & & & \\
Coherent noise & - & - & 0 & 0 \\
$\quad$ Azimuth (deg) & 0 & - & 90 & 90 \\
Inclination (deg) & 0 & 0 & 0.5 & 0.66 \\
Energy & & & & 0 \\
$\quad$ Time delay (msec) & 0.06 & 0.06 & 0.06 & 0.06 \\
Random noise & 0.06 & 0.06 & 0.06 & 0.06 \\
$\quad$ Energy (north) & 0.06 & 0.5 & 0.06 & 0.5 \\
$\quad$ Energy (east) & 5.5 & 1.6 & 1.5 & 0.8 \\
$\quad$ Energy (vertical) & & & \\
Signal-to-noise energy ratio & & & & \\
\hline
\end{tabular}

The signal-to-noise values (SNR) are not derived using equations (9) or (4), but obtained directly from the synthetic data specifications in this table. 
Table 2

True Error, Linearity $\left(\operatorname{lin}_{j}\right)$, and Confidence Estimate $\left(\varepsilon_{95_{j}}\right)$ for $P$-Wave Polarization Estimates, Obtained Using Five Alternative Measuring Techniques on Synthetic Data

\begin{tabular}{|c|c|c|c|c|c|}
\hline & & \multicolumn{4}{|c|}{ Station Number } \\
\hline & & 1 & 2 & 3 & 4 \\
\hline \multirow{3}{*}{$\begin{array}{l}3 \mathrm{C} \text { complex SVD (1) } \\
\text { (no weighting) }\end{array}$} & Error (deg) & 0 & 14.5 & 17 & 24.5 \\
\hline & $\operatorname{lin}_{j}(\%)$ & 99.9 & 99.8 & 99.5 & 96.1 \\
\hline & $\varepsilon_{95_{j}}(\mathrm{deg})$ & 2.5 & 4 & 3 & 2.5 \\
\hline \multirow{3}{*}{$\begin{array}{l}3 \mathrm{C} \text { complex SVD (2) } \\
\text { (with weighting) }\end{array}$} & Error (deg) & 0 & 0 & 17 & 16.5 \\
\hline & $\operatorname{lin}_{j}(\%)$ & 99.9 & 99.8 & 99.4 & 94.4 \\
\hline & $\varepsilon_{95_{j}}(\mathrm{deg})$ & 2.5 & 5 & 3 & 4 \\
\hline \multirow{3}{*}{$\begin{array}{l}4 \times 3 \mathrm{C} \text { complex SVD (3) } \\
\text { (with weighting) }\end{array}$} & Error (deg) & 0 & 0 & 9.5 & 6.5 \\
\hline & $\operatorname{lin}_{j}(\%)$ & 99.3 & 99.2 & 96.6 & 85.8 \\
\hline & $\varepsilon_{95_{j}}(\operatorname{deg})$ & 3.5 & 5 & 4 & 4.5 \\
\hline \multirow{3}{*}{$\begin{array}{l}3 \mathrm{C} \text { complex SVD (4) } \\
\text { (with weighting and optimized window) }\end{array}$} & Error (deg) & 0 & 0 & 2 & 16 \\
\hline & $\operatorname{lin}_{j}(\%)$ & 99.9 & 99.8 & 99.6 & 94.2 \\
\hline & $\varepsilon_{95_{j}}(\mathrm{deg})$ & 2.5 & 5.5 & 4 & 4 \\
\hline \multirow{3}{*}{$\begin{array}{l}4 \times 3 \mathrm{C} \text { complex SVD }(5) \\
\text { (with weighting and optimized window) }\end{array}$} & Error (deg) & 0 & 0 & 0 & 8.5 \\
\hline & $\operatorname{lin}_{j}(\%)$ & 98.4 & 98.2 & 98.4 & 87.6 \\
\hline & $\varepsilon_{95_{j}}(\mathrm{deg})$ & 3.5 & 6.5 & 4 & 6 \\
\hline
\end{tabular}

The reported values are the averages of 200 realizations using the synthetic data described in Figure 3 and Table 1. Each confidence interval defines the opening angle of the confidence cone that has the estimated polarization as axis. The estimates with window optimization relate to a sample misfit acceptance interval of $\left[-\mu_{90},+\mu_{90}\right]$ and a minimum number of 30 samples in the optimized window.

The next synthetic test investigates the working of our method in the presence of relative arrival-time errors between stations. This is to simulate the case where no crosscorrelation data alignment is applied, or where the data do not allow a robust arrival-time alignment. We use the same data as in the previous test, but impose random arrival-time perturbations at each station. These perturbations have standard deviations of respectively, $0 \mathrm{sec}, 1 \mathrm{sec}, 2 \mathrm{sec}, 3 \mathrm{sec}$, $4 \mathrm{sec}$, and $5 \mathrm{sec}$. A 5 -sec arrival-time error at individual stations translates in a 10 -sec relative timing error between stations. This is significant in relation to the $33 \mathrm{sec}(30 \mathrm{~Hz})$ dominant wavelength of the synthetic data.

Figure 4 shows how, for each station, the polarization errors, confidence estiamtes $\varepsilon_{95_{j}}$, and linearities $\operatorname{lin}_{j}$ respond to increasing arrival-time errors. These average estimates and their error bars are calculated from 200 realizations with different added random background noise. In general, the figure indicates a gradual reliability deterioration of polarizations and their quality estimates with increasing arrivaltime errors. Mistimings are especially problematic in stations with correlated noise. This is evident from the changes in polarization errors at stations 3 and 4 . Contrary to the linearities $l i n_{j}$, we find that the confidence estimates $\varepsilon_{95_{j}}$ vary only slightly. Timing errors with standard deviations as low as $3 \mathrm{msec}$ cause the linearities at stations 1 to 3 to drop from $98.5 \% \pm 1 \%$ to $90 \% \pm 10 \%$. At station 4 the linearities drop from $87.5 \% \pm 2 \%$ to $80 \% \pm 20 \%$. This test indicates that an accurate arrival-time correction is vital to the proper working of our method. When such accurate time alignment is impossible the quality estimates, especially linearities, will nevertheless indicate that polarization estimates are unreliable. Linearities provided by our polarization analysis are therefore especially useful to identify stations with poor data quality and/or poor arrival-time alignment.

\section{Real Data Example}

Dataset. The real data used for testing our automated approach comes from a passive seismic-monitoring experiment that was conducted in the Valhall oil field in 1998. This field is situated in the Norwegian sector of the Central North Sea Graben. The reservoir rock is a mechanically weak and highly porous chalk that is compacting in response to production. This compaction has lead to seafloor subsidence of up to $4 \mathrm{~m}$ and is considered the main reason for microseismic activity within the reservoir overburden (Dyer et al., 1999). Between 2 June and 26 July 1998 a CGG-SST500, six-level, $3 \mathrm{C}, 30-\mathrm{Hz}$ geophone string was installed in well $2 / 8-\mathrm{A} 3 \mathrm{~B}$. The geophone stations were deployed at 20-m intervals between depths of 2000 and $2100 \mathrm{~m}$, just above the reservoir and are numbered from 1 to 6 with increasing depth (Fig. 5). The data have a sampling rate of $1000 \mathrm{~Hz}$. A total of 303 locatable events were recorded during the experiment (Dyer and Jones, 1998). The locations of these events are calculated using a local velocity model, the $P$-wave and $S$-wave arrival times, and the $P$-wave polarizations at each station along the vertical array. Because the Valhall experiment uses a linear vertical array, the $P$-wave polarizations serve as es- 

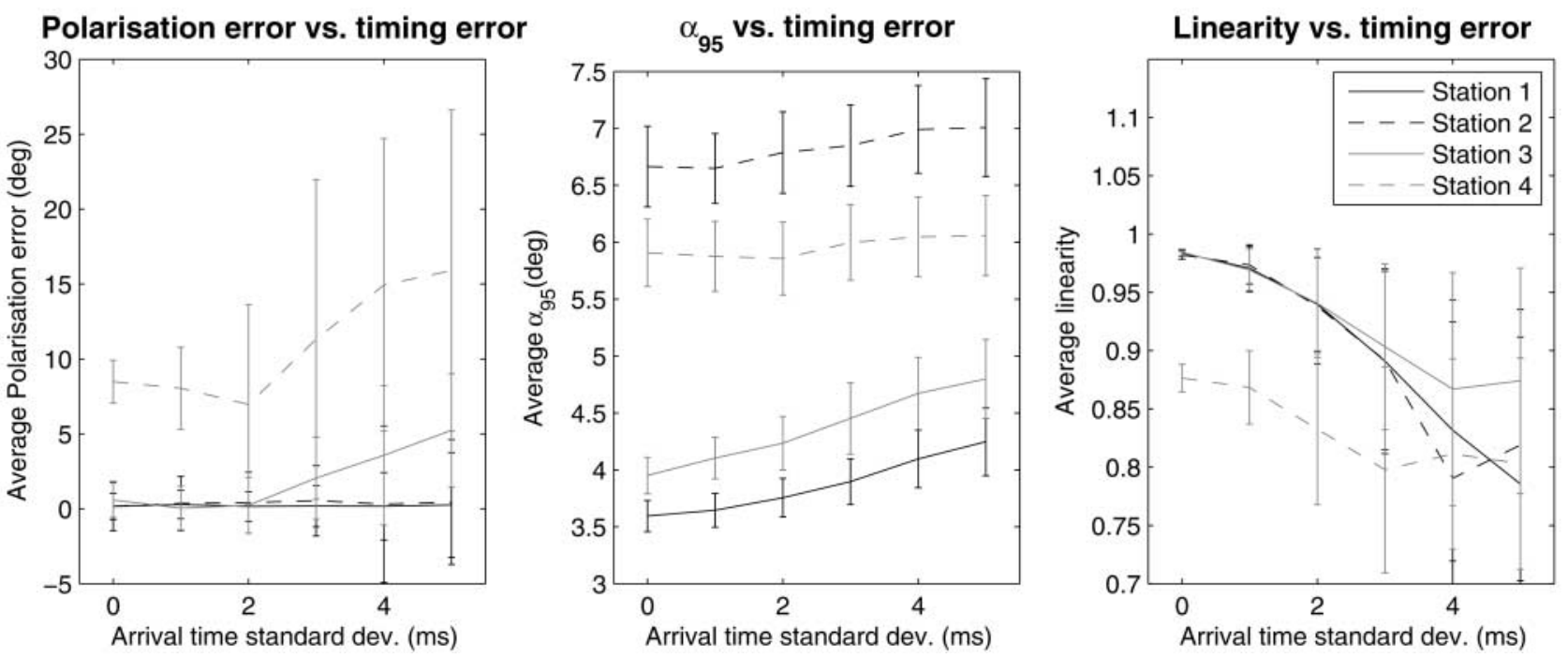

Figure 4. Results of a test that investigates the performance of our technique in the presence of arrival time errors between stations. The horizontal axis indicates the standard deviation of the random arrival time perturbation at individual stations. The reported values for polarization error, linearity, and confidence intervals are the averages of 200 realizations using the synthetic data described in Figure 3 and Table 1. The error bars relate to half the standard deviation of the average values.

timates for the source-receiver direction. They are absolutely vital to adequately constrain the source locations. In early work the $P$-wave polarizations were obtained manually using a single station, unweighted EVD on raw $P$-wave data (Dyer et al., 1999). We use these results as a benchmark.

The overall noise polarization properties are investigated using prearrival data. For the top four stations we found that the noise is predominantly isotropically polarized. The bottom station, and station 5 to a lesser extent, experienced 5 to 10 times higher levels of mainly horizontally polarized noise. This noise is thought to be related to the unclamped weight, attached to the lowermost geophone station (R. Jones, personal comm.). The signal and noise both have typical bandwidths between $5 \mathrm{~Hz}$ and $60 \mathrm{~Hz}$ and peak at $30 \mathrm{~Hz}$. As a consequence, no improvement in signal-tonoise ratio by bandpass filtering the data was expected and no filter was therefore applied. A few events and stations were checked for the presence of coherent (signal-generated) noise in the $P$-wave coda. Such noise was found in several records. The $P$ wave and $S$ wave are separated sufficiently in time $(0.5 \mathrm{sec})$ such that the noise on the $P$ wave is mainly due to scatter and multipathing effects. The presence of both polarized random and coherent noise makes this dataset ideal to test our automated polarization analysis.

Implementation and Results. First, accurate relative $P$ wave arrival times are calculated by repeated cross-correlation with pilot traces (see Practical Implementation on $P$ Wave Array Data section). The manual arrival times of Dyer and Jones (1998) served as a starting point.

For the automatic $P$-wave polarization analysis the length of the initial analysis window was set to $60 \mathrm{msec}$, starting with the $P$-wave arrival time. This length was chosen after visual inspection of the data and comprises approximately two signal periods. We judged that a minimal number of 25 samples for the optimized analysis window is sufficient to provide a representative polarization estimate. An $\alpha$-value of $90 \%$ is used in the window optimization to remove all samples with misfit values $\gamma(t)$ that lie outside the expectancy interval $\left[-\mu_{\alpha},+\mu_{\alpha}\right]$. The weighting matrix $\mathbf{W}$ was calculated from $300 \mathrm{msec}$ of prearrival data. All stations with linearities, $\mathrm{lin}_{j}$, of less than $95 \%$, or with a polarization uncertainty estimate, $\left|\varepsilon_{95}\right|$, greater than $6^{\circ}$ were deemed unreliable and subsequently discarded.

A total of 1463 polarization estimates were obtained using these parameters. Figure 6 displays the results for a representative microseismic event. It contains the original data for all six stations and the associated automatically determined $P$-wave polarizations. On hodograms, or particle motion plots, for stations 1,2 , and 5 there is clear evidence that the $P$-wave particle motion becomes nonlinear after approximately half a period. Station 4 has seemingly the best data quality, whereas station 6 suffers from increased background noise. The automatically interpreted polarizations are in good agreement with the general $P$-wave particle motion trends.

In Figure 7 we show the histograms of the polarization uncertainty estimates $\left|\varepsilon_{95_{j}}\right|$ for each station $j$. These polarization uncertainty estimates have slightly asymmetric distributions and range between $0.5^{\circ}$ and $6^{\circ}$. The shape and spread of these uncertainty distributions confirm that the threshold of $6^{\circ}$ for identifying bad estimates is appropriate for this dataset. The median of the uncertainty estimates decreases from $3^{\circ}$ in station 1 to $2.1^{\circ}$ in station 4 . Values then rise to 


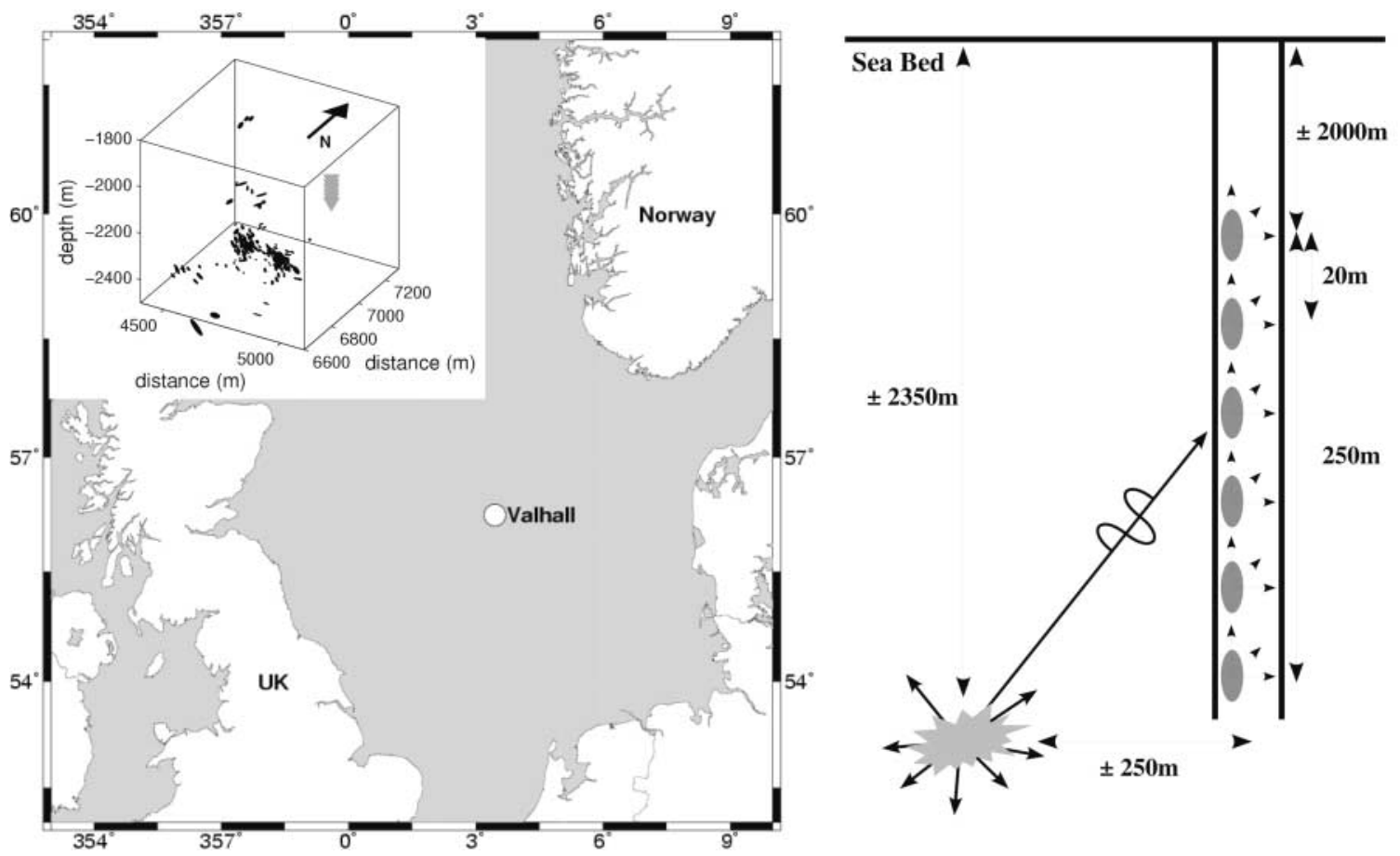

Figure 5. (Left) Valhall location map with 3D inset reporting the locations of the microearthquake sources recorded during a 1998 experiment and after Dyer et al. (1999). (Right) General geometry overview of the 1998 experiment. The geophone stations were deployed at 20-m intervals between depths of 2000 and $2100 \mathrm{~m}$ and are numbered from 1 to 6 with increasing depth.

$3.4^{\circ}$ in stations 5 and 6 . Station 1 is furthest away from the cluster of microseismic events and has lower overall signalto-noise levels because of longer travel paths. This explains the progressively decreasing uncertainty estimates found in stations $1,2,3$, and 4 . This trend is not continued in results from stations 5 and 6 because of increased noise from the unclamped weight beneath station 6 .

\section{Comparison with Manually Determined Polarizations.}

The accuracy and quality of our automatically obtained polarization estimates is finally assessed by comparing them with the manual polarizations from Dyer and Jones (1998). We analyze the scatter of our polarizations with respect to the benchmark ones. The scatter estimate is independent of the range of polarizations and is evaluated per station and plotted in stereographic projection. First, we take the manually obtained reference polarization for a given station and event, and rotate it through azimuth and inclination to the vertical, or pole (points A and B to a and b in Fig. 8). The same rotation is applied to the relevant polarization from our set of estimates (points $\mathrm{A}^{\prime}$ and $\mathrm{B}^{\prime}$ to $\mathrm{a}^{\prime}$ and $\mathrm{b}^{\prime}$ in Fig. 8). All reference polarizations lie on the pole after rotation and our polarizations scatter around it. An overall deviation of our rotated polarizations from the pole indicates a systematic difference between both sets of estimates. Scatter in the north-south direction, such as between $\mathrm{b}$ and $\mathrm{b}^{\prime}$, relates to inclination differences between the two sets of polarizations.
Scatter in the east-west direction, such as between a and $\mathrm{a}^{\prime}$, relates to azimuth differences (Fig. 8).

We determine the centre of mass of our rotated polarization estimates and its $95 \%$ expectancy limits $\max _{95}$ and $\min _{95}$ (Fisher et al., 2004). If the differences between our and the manually obtained reference estimates of Dyer and Jones (1998) are perfectly random then the angle between the center of mass and the pole is zero and $\max _{95}=\min _{95}$. Ideally, the values for $\max _{95}$ and $\min _{95}$ should also be similar to the estimated confidence limits on our polarizations $\varepsilon_{\alpha_{j}}$.

The set of measurements from Dyer and Jones (1998) contain 1325 determined $P$-wave polarizations, 1228 of which are also measured by using our automated approach and can thus be compared. A stereographic projection for the scatter between both sets of polarization estimates in each station is given in Figure 9. The number of compared estimates per station and the angle between the pole and the centre of mass of the rotated estimates with the $95 \%$ expectancy intervals are given in Table 3.

Except for station 5, we find trivial deviation angles between the pole and the scatter center of gravity. For station 5 we have an average deviation of $3^{\circ}$ to the east. The scatter, moreover, is elongated between $2^{\circ} \mathrm{W}$ and $10^{\circ} \mathrm{E}$. This indicates that our 223 compared polarization azimuths are shifted azimuthally clockwise with respect to the benchmark ones. The most plausible reason for this bias is increased horizontal noise levels due to the unclamped weight below 
Data
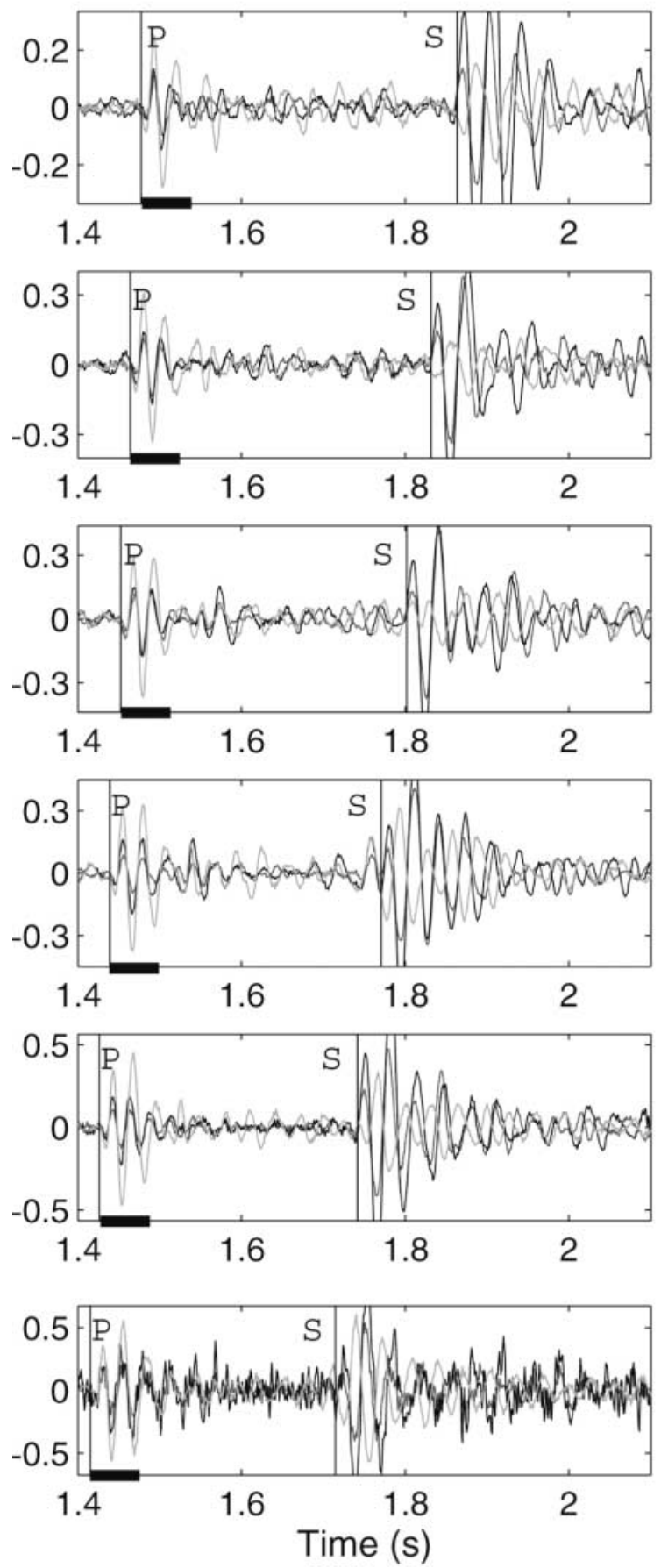

Hodogram horizontal plane
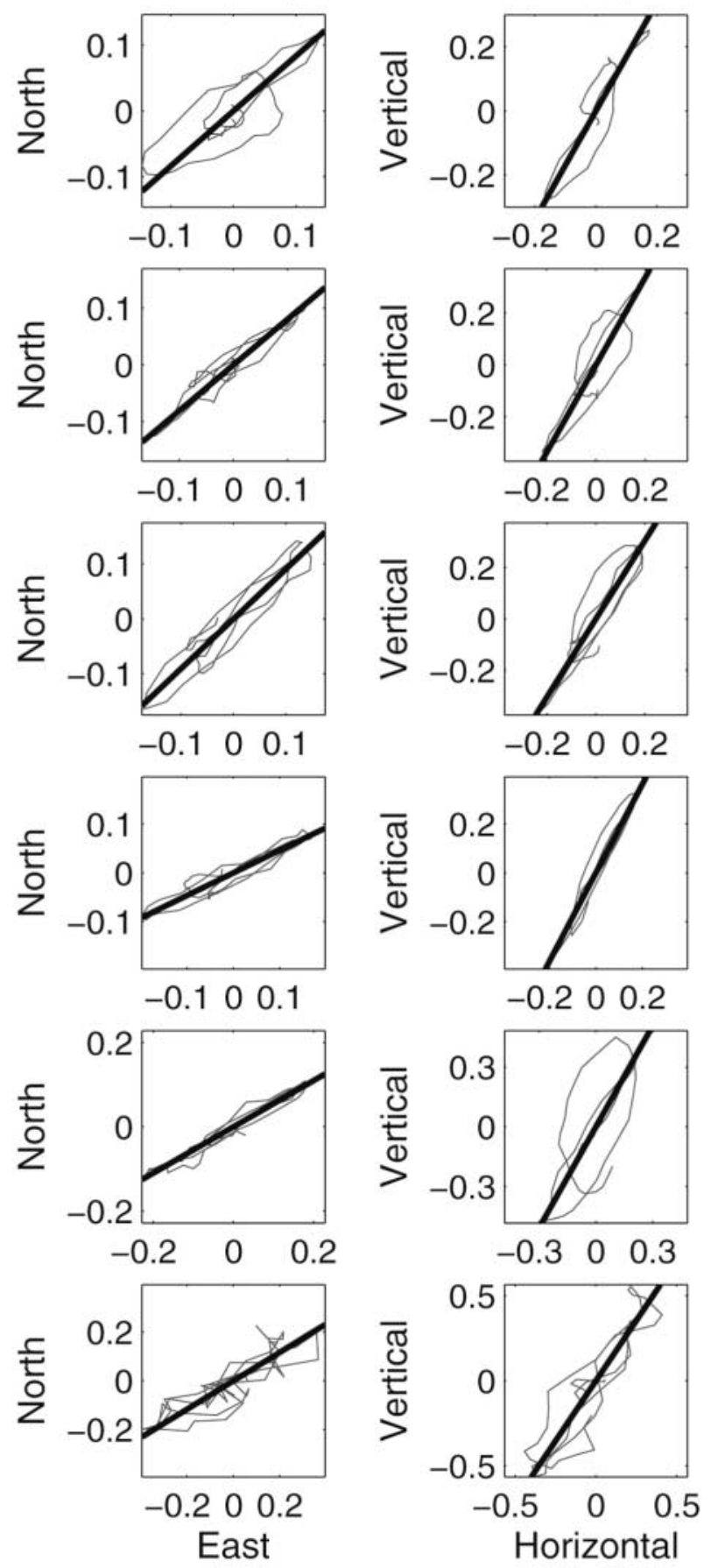

Figure 6. The left panels shows an example of a microseismic event recorded at Valhall. The receivers are numbered 1 to 6 from top to bottom. The $P$-wave and $S$ wave arrivals are picked on the $3 \mathrm{C}$ data from each station. The horizontal bold lines that start at the $P$-wave arrivals mark the initial time windows used for polarization analysis. The left panel shows the horizontal and vertical hodograms, or particle motion plots, for each station. They represent the $60 \mathrm{msec}$ of data as indicated by the bold markers on the left panels. These hodograms are overlain with the automatically measured $P$-wave polarizations. 

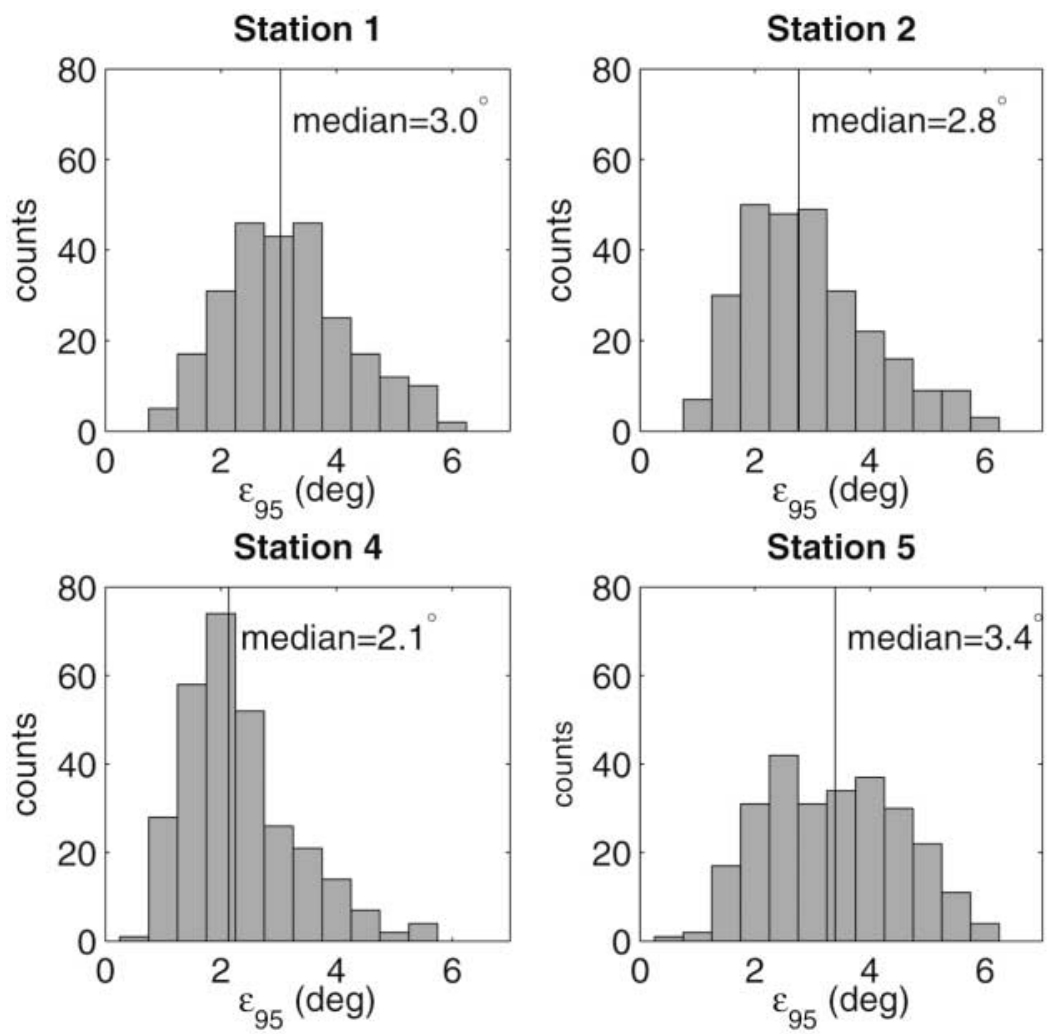

Station 5

Station 2

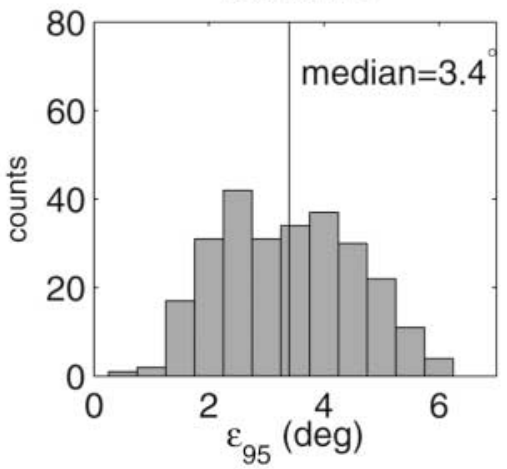

Station 3

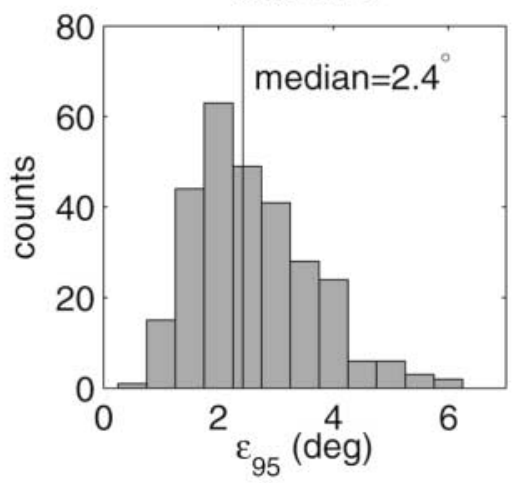

Station 6

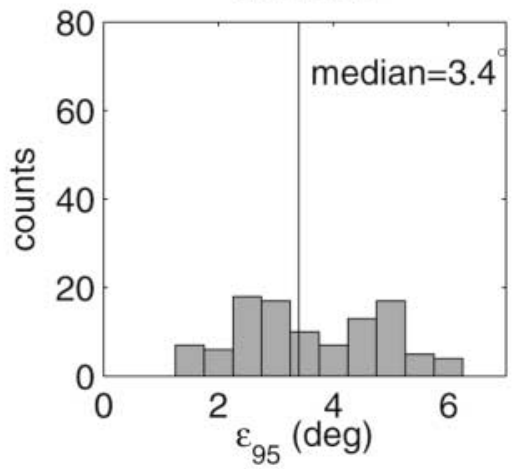

Figure 7. Histograms per station for the polarization uncertainty estimates, $\varepsilon_{95_{j}}$. The polarization uncertainties gradually decrease from stations 1 to 4 . They are largest for stations 5 and 6, probably because of the presence of an unclamped weight attached to station 6 .

station 6. The observed azimuthal shift in polarizations is then expected, since our polarization analysis corrects for noise polarization effects whereas the method for the benchmark polarizations does not. For the same reason we would anticipate a more pronounced effect at station 6 . This is not observed because noise from the undamped weight rendered most data from this station inadequate for analysis. The 63 compared polarization estimates at this station relate mainly to quiet times. Therefore, we do not expect a systematic shift in azimuths as is the case.

Ninety-five percent of the compared automatically obtained polarization estimates differ by less than $4^{\circ}$ from the manual polarizations from Dyer and Jones (1998). This is supported by the expectancy intervals $\max _{95}$ and $\min _{95}$ for differences between both sets of polarizations (Table 3). At most stations we find small and similar values for $\max _{95}$ and $\min _{95}$ values. This indicates that the differences between both sets of polarizations are mainly due to random picking errors. Finally, we observe a good agreement between $\max _{95}$ and $\min _{95}$ values and estimated confidence intervals $\varepsilon_{95_{j}}$ for the automatic polarizations. This gives us an increased confidence in the automatically derived error estimates.

\section{Discussion}

Our noise-weighted 3C array method for polarization analysis and signal extraction uses analytic signals as input data to analyze signals with elliptical and linear polarizations (Vidale, 1986). The input matrices could also consist of time series, or their Fourier transforms or wavelet transforms (Flinn, 1965; Samson, 1973; Lilly and Park, 1995). We prefer the analytic signal because it is computationally more cost effective than other transforms. In addition, it is stable over short-analysis windows which allows the removal of individual samples from the data window. Fourier transforms, and especially wavelet transforms are nevertheless better suited for broadband data where signal and noise separate in the frequency domain. Noise weighting is included to deal with the bias introduced by polarized noise. It is an important feature for data with low signal-to-noise ratios and strongly polarized noise (Souriau and Veinante, 1975; Bataille and Chiu, 1991). Weighting relies on prior knowledge of the noise-correlation matrix and the assumption of stationary-noise properties. Typically, the weighting matrix is estimated from 5 to 10 signal periods of prearrival data (Samson, 1983a; Du et al., 2000). The method is robust for 


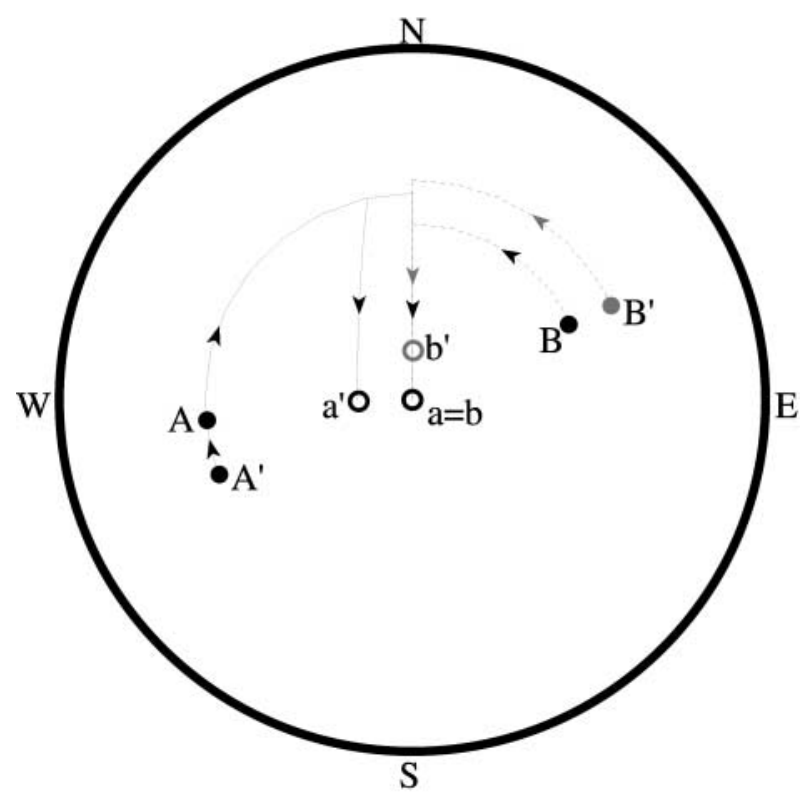

Figure 8. Method to compare our polarizations with the reference polarizations in stereographic projection. A and B (in black) are two reference polarizations. $\mathrm{A}^{\prime}$ and $\mathrm{B}^{\prime}$ (in dark and light gray) are our alternative estimates for $\mathrm{A}$ and $\mathrm{B}$. We move $\mathrm{A}$ and $\mathrm{B}$ to the pole, creating points $\mathrm{a}$ and $\mathrm{b}$, first by rotating them horizontally to an azimuth of $0^{\circ}$ and then vertically to an inclination of $90^{\circ}$. Next, the same rotations are applied, respectively, to our polarization estimates $\mathrm{A}^{\prime}$ and $\mathrm{B}^{\prime}$, thereby creating the points $\mathrm{a}^{\prime}$ and $b^{\prime}$ close to the pole. The scatter of points $a^{\prime}$ and $b^{\prime}$ around the pole defines the difference between our polarizations and the reference ones. Scatter in the east-west direction ( $a$ and $a^{\prime}$ ) relates to differences in azimuth. Scatter in the north-south direction (b and $\left.b^{\prime}\right)$ relates to differences in inclination.

small errors in the weighting matrix. To verify if weighting improves the estimates the signal-to-noise ratio $\mathrm{SNR}^{w}$ (9) must be greater than its unweighted counterpart SNR (4).

To improve accuracy in the presence of signal-correlated noise we combine data from different $3 \mathrm{C}$ stations into one analysis (Bear et al., 1999). The method only works when all data relate to the same signal. The data must also be accurately corrected for arrival-time differences. When the noise is unrelated between $k$ combined datasets, the signal and polarization estimates will benefit from a $\sqrt{k}$-fold increase in signal-to-noise. The main advantage over stacking like Jurkevics (1988), or Earle (1999), is that this multistation approach provides signal and polarization estimates at each station and that the signal polarizations and amplitudes may vary from station to station.

Our automated procedure for $P$-wave polarization analysis relies on several parameters. The first set of parameters control the optimization of the analysis window. It includes a prearrival data window for noise weighting and the initial $P$-wave window. These are typically 5 to 10 , and 2 signal periods long. We find that reasonable changes in window lengths have little influence on the final result. The $\alpha$-value that defines the misfit expectancy interval $\left[-\mu_{\alpha},+\mu_{\alpha}\right]$, and the minimum number of samples in the optimized analysis window are more influential. For $\alpha<85 \%$ the window optimization nearly always converges to the predefined minimum number of samples. We obtained best results with $\alpha$ values of approximately $90 \%$ and a minimal sample population of approximately 0.5 to 1 signal period. The second set of parameters are the threshold values for linearity $\operatorname{lin}_{j}$ and uncertainty $\varepsilon_{\alpha_{j}}$. They control the identification of events and stations with poor data quality. This ultimately leads to the removal of low-quality data from the analysis. We feel that linearity $\operatorname{lin}_{j}$ should always exceed 95\% and that the error estimate should be less than $10^{\circ}$. When this is not the case the polarization estimate should be regarded as unreliable.

\section{Conclusions}

We introduce a complex, weighted, 3C-array method to determine the waveform and polarization of the recorded seismic signal at each 3C station. Our method is based on SVD, or EVD, of the analytic signal and combines approaches from Vidale (1986), Samson (1983a), and Bear and Pavlis (1999). It can handle linear and elliptically polarized signals and minimises the influence of polarized noise on the estimated signal properties.

We have combined this method with an optimization procedure for selecting the data-analysis window and adapt it for $P$-wave polarization analysis. It automatically detects and removes stations with poor-quality data for each event. It also provides a $P$-wave polarization with error and linearity estimates. Tests on synthetic and real data show that the combination of linearity and a confidence interval of errors accurately indicate the quality of the $P$-wave polarization estimate.

We implemented our fully automated method on the 1998 Valhall microseismic dataset and obtained $1463 P$ wave polarizations estimates from 303 events and 6 receiv-

Table 3

Measured Differences between the Reference and Our $P$-Wave Polarization Estimates at Each Station, Based on a EVD of the Rotated Polarization Vectors Shown in Figure 9

\begin{tabular}{lcccccc}
\hline & \multicolumn{7}{c}{ Station Number } \\
\cline { 2 - 7 } & 1 & 2 & 3 & 4 & 5 & 6 \\
\hline $\max _{95}(\mathrm{deg})$ & 4.6 & 4.6 & 5 & 3.7 & 5.6 & 3.2 \\
$\min _{95}(\mathrm{deg})$ & 3.5 & 3.6 & 3.8 & 3.1 & 2.2 & 2.7 \\
$\begin{array}{c}\text { Deviation from vertical of } \\
\quad \text { overall difference (deg) }\end{array}$ & 1 & 0.5 & 1 & 1 & 3 & 0.5 \\
No. of estimates & 194 & 222 & 254 & 255 & 223 & 63 \\
\hline
\end{tabular}

Large angles between the pole and the first eigenvectors indicate systematic differences between our polarizations and the reference polarizations. $\max _{95}$ and $\min _{95}$ give the minimum and maximum $95 \%$ expectancy intervals for the difference between original and automatic polarizations. 

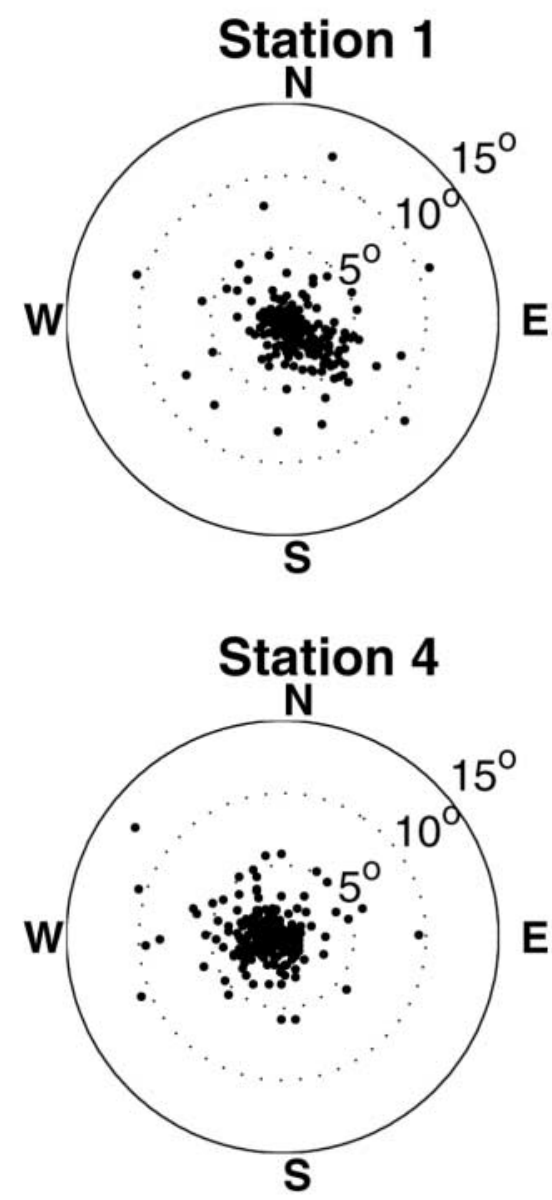
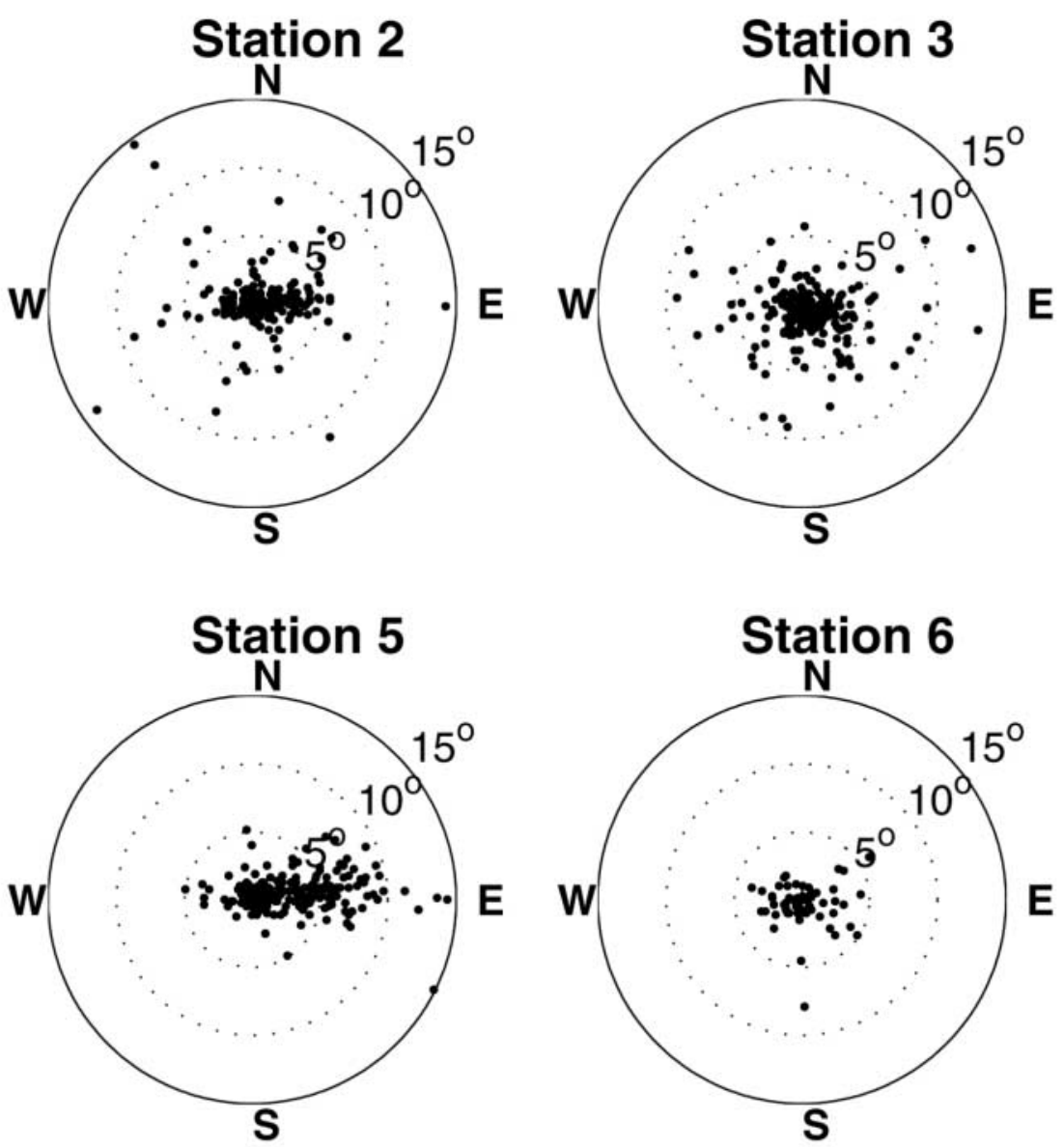

Figure 9. Stereographic projections of the scatter between our polarization estimates and the reference polarization estimates from Dyer and Jones (1998). Each scatter plot is generated as explained in Figure 8. Except for station 5, no systematic difference occurs between the manual and reference polarizations as the scatter is typically less than $5^{\circ}$.

ers. These estimates typically have $95 \%$ confidence limits of $3^{\circ}$. We statistically compared our results with those from a previous study by Dyer et al. (1999). The reference $P$-wave polarizations were obtained by applying a simple, singlestation EVD over a manually selected data window. The difference between both sets of polarizations is mainly due to random picking errors. $95 \%$ of all polarization differences lie within $4^{\circ}$ and none exceed $15^{\circ}$. This confirms that our automated method provides meaningful error estimates and polarizations that are of comparable quality to manual measurements. An additional advantage is naturally that the automated analysis takes only a few minutes for the entire dataset, compared with a time-intensive manual interpretation.

A significant subset of events in receiver station 5 are contaminated with horizontally polarized noise which causes a shift of up to $10^{\circ}$ in the azimuths from the reference polarizations in Dyer and Jones (1998). Our technique corrects for this bias and therefore leads to more accurate polarization estimates. In turn this improves the quality of the estimated event locations.

\section{Acknowledgments}

The authors would like to thank ABB Offshore Systems, BP Norge, Shell Exploration and Production UK and Schlumberger Cambridge Research for funding this research through the Leeds Microseismicity Project. We are grateful to A.B.B. and B.P. for providing the data and to Rob Jones for providing the initial suggestion for this work. We greatly appreciate one anonymous reviewer and Robert de Franco for their helpful comments and suggestions.

\section{References}

Bataille, K., and J. Chiu (1991). Polarization analysis of high-frequency, three-component seismic data, Bull. Seism. Soc. Am. 81, no. 2, 622642 .

Bear, L., and G. Pavlis (1999). Multi-channel estimation of time residuals from broadband seismic data using multi-wavelets, Bull. Seism. Soc. Am. 89, no. 3, 681-692.

Bear, L., G. Pavlis, and G. Bokelmann (1999). Multi-wavelet analysis of three-component seismic arrays: Application to measure effective anisotropy at Piñon Flats, California, Bull. Seism. Soc. Am. 89, no. 3, 693-705.

Butler, R. (1992). Statistics of paleomagnetic data, in Paleamagnetism: 
Magnetic Domains to Geologic Terranes, Blackwell Scientific Publications, Malden, Massachusetts.

Claassen, J. (2001). Robust bearing estimation for three-component stations, Pure Appl. Geophys. 158, 349-374.

de Franco, R., and G. Musacchio (2001). Polarization filter with singular value decomposition, Geophysics 66, no. 3, 932-938.

Du, Z., G. Foulger, and W. Mao (2000). Noise reduction for broad-band, three-component seismograms using data-adaptive polarization filters, Geophys. J. Int. 141, 820-828.

Dyer, B., and R. Jones (1998). 3D processing and interpretation of the microseismic data recorded during June and July 1998 in the Valhall Field, Technical Report IR03/82, CSM Associates LTD.

Dyer, B., R. Jones, J. Cowles, O. Barkved, and P. G. Folstad (1999). Microseismic survey of a North Sea reservoir, World Oil 220, no. 3, 74-78.

Earle, P. (1999). Polarization of the Earth's teleseismic wavefield, Geophys. J. Int. 139, 1-8.

Fisher, N., T. Lewis, and B. Embleton (2004). Statistical Analysis of Spherical Data, Cambridge University Press, New York, 160-178.

Flinn, E. (1965). Signal analysis using rectilinearity and direction of particle motion, Proc. IEEE 53, 1874-1876.

Jurkevics, A. (1988). Polarization analysis of three-component array data, Bull. Seism. Soc. Am. 78, no. 5, 1725-1743.

Lilly, M., and J. Park (1995). Multiwavelet spectral and polarization analyses of seismic records, Geophys. J. Int. 122, 1001-1021.

Mao, W., and D. Gubbins (1995). Simultaneous determination of time delays and stacking weights in seismic array beamforming, Geophysics 60, no. 2, 491-502.

Maxwell, S., R. Young, R. Busso, A. Jupe, and J. Dangerfield (1998). Microseismic logging of the Ekofisk reservoir, SPE/ISRM 47276.

Montalbetti, J., and E. Kanasewich (1970). Enhancement of teleseismic body phases with a polarization filter, Geophys. J. R. Astr. Soc. 21, 119-129.

Reading, A., W. Mao, and D. Gubbins (2000). Polarization filtering for automatic picking of seismic data and improved converted wave detection, Geophys. J. Int. 147, 227-234.

Rowe, C., R. Aster, W. Philips, R. Jones, B. Borchers, and M. Fehler (2002). Using automated, high-precision repicking to improve delin- eation of microseismic structures at the Soultz geothermal reservoir, Pure Appl. Geophys. 159, 563-596.

Samson, J. (1973). Description of the polarization states of vector processes: applications to ULF magnetic fields, Geophys. J. R. Astr. Soc. 34, 403-419.

Samson, J. (1983a). Pure states, polarized waves, and principal components in the spectra of multiple, geophysical time-series, Geophys. J. R. Astr. Soc. 72, 647-664.

Samson, J. (1983b). The spectral matrix, eigenvalues, and principal components in the analysis of multichannel geophysical data, Ann. Geophys. 1, 115-119.

Samson, J., and J. Olsen (1980). Some comments on the description of the polarization states of waves, Geophys. J. R. Astr. Soc. 61, 115-129.

Schimmel, M., and J. Gallart (2003). The use of instantaneous polarization attributes for seismic signal detection and image enhancement, Geophys. J. Int. 155, 653-668.

Schulte-Pelkum, V., G. Masters, and P. Shearer (2001). Upper mantle anisotropy from long-period $P$ polarization, J. Geophys. Res. 106, no. B10, 21,917-21,934.

Silver, P., and W. Chan (1991). Shear-wave splitting and subcontinental mantle deformation, J. Geophys. Res. 96, 16,429-16,454.

Souriau, M., and J. Veinante (1975). Three adaptive filters for the detection of body waves, with application to deep seismic soundings, Bull. Seism. Soc. Am. 65, no. 4, 995-1012.

Teanby, N., J.-M. Kendall, R. Jones, and O. Barkved (2004). Stress-induced temporal variations in seismic anisotropy observed in microseismic data, Geophys. J. Int. 156, 459-466.

Vidale, T. (1986). Complex polarization analysis of particle motion, Bull. Seism. Soc. Am. 76, no. 5, 1393-1405.

School of Earth and Environment

Earth Science Building

University of Leeds

Leeds, LS2 9JT United Kingdom

Manuscript received 16 November 2005. 